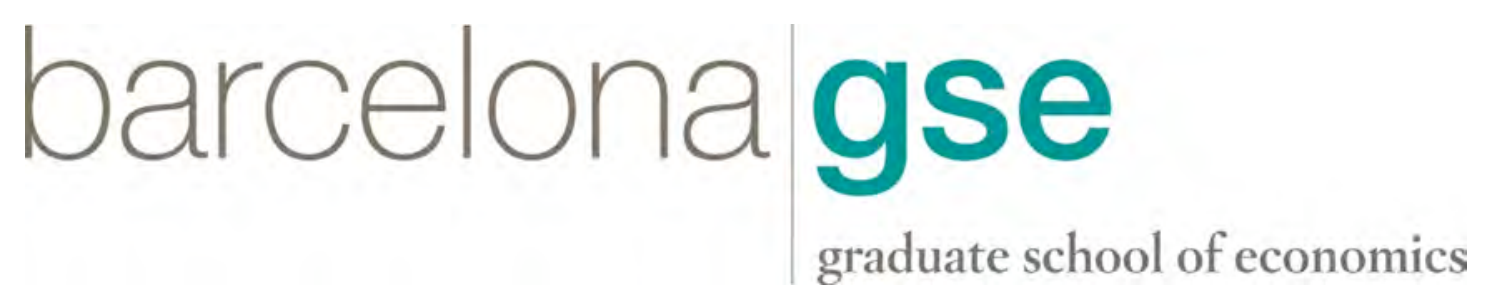

\title{
Unemployment, Participation and Worker Flows Over the Life-Cycle
}

\author{
Sekyu Choi \\ Alexandre Janiak \\ Benjamín Villena-Roldán
}

March 2012

Barcelona GSE Working Paper Series

Working Paper $n^{\circ} 617$ 


\title{
Unemployment, Participation and Worker Flows Over the Life-Cycle *
}

\author{
Sekyu Choi ${ }^{1}$, Alexandre Janiak² , and Benjamín Villena-Roldán ${ }^{3}$ \\ ${ }^{1}$ Universitat Autònoma de Barcelona, Barcelona GSE and MOVE \\ ${ }^{2}$ Universidad de Chile, Center for Applied Economics \\ ${ }^{3}$ Universidad de Chile, Center for Applied Economics
}

March 2012

\begin{abstract}
We estimate and report life-cycle transition probabilities between employment, unemployment and inactivity for male and female workers using Current Population Survey monthly files. We assess the relative importance of each probability in explaining the life-cycle profiles of participation and unemployment rates using a novel decomposition method. A key robust finding is that most differences in participation and unemployment over the life-cycle can be attributed to the probability of leaving employment and the probability of transiting from inactivity to unemployment, while transitions from unemployment to employment (the job finding probability) play secondary roles. We conclude that search models that seek to explain life-cycle work patterns should not ignore transitions to and from inactivity.
\end{abstract}

Keywords: Life-cycle, Unemployment, Participation, Worker flows.

\section{JEL Codes: D91, E24, J64}

*email: sekyu.choi@uab.cat, ajaniak@dii.uchile.cl, bvillena@dii.uchile.cl. We would like to thank Philipp Kircher, Omar Licandro, Morten Ravn, Robert Shimer, Thijs van Rens, Ludo Visschers, seminar participants at the SED, ESEM, LAMES, SECHI, VI REDg Workshop at UAB, PUC-Chile, Warwick and Manchester and our colleagues at UAB and CEA for helpful discussions. Sekyu Choi gratefully acknowledges financial support from the Spanish Ministry of Education through grant ECO2009-09847. Alexandre Janiak and Benjamin Villena thank Fondecyt for financial support (project no 1120593). All errors are ours. 


\section{Introduction}

Labor market experiences are significantly different over the life-cycle: unemployment rates are higher for younger individuals while participation rates fall dramatically for workers after certain ages. There is also evidence of heterogeneous labor supply volatilities by age groups, as noted by Blanchard and Diamond (1990), Rios-Rull (1996) and Jaimovich and Siu (2009). However, little is known about the worker flows involved in this process: Is high unemployment among the young the result of high job separation or low job finding probabilities? Are transitions in and out of the labor force important?

In this paper we explore the dynamics of transition probabilities between employment, unemployment and inactivity over the life-cycle, using Current Population Survey (CPS) monthly data for male and female workers. ${ }^{1}$ We construct measures of worker flows between three labor force states (employment, unemployment and inactivity). From this procedure we can estimate age-dependent job finding, separation, and labor force exit and entry probabilities.

We propose a methodology to account for the relative importance of each of these transition probabilities in shaping the life-cycle profiles of unemployment and participation rates. We simulate the labor status of a cohort of individuals by using age-dependent Markov chains estimated from the CPS monthly data. In this way, we can compare the empirical participation and unemployment profiles with those obtained by our simulations when shutting down life-cycle heterogeneity of a subset of transition probabilities.

We find that most differences in participation and unemployment rates over the life-cycle can be attributed to the probability of leaving employment and the probability of transiting from inactivity to unemployment. These results hold for both male and female workers. However, transitions from unemployment to employment play only minor roles. Higher probability of leaving employment at the beginning of the life-cycle is important to explain the differences in unemployment and participation stocks between young and workers aged above 30. Moreover, the decrease in the probability of transiting from inactivity to unemployment at the end of the life-cycle is relevant to account for the increase in inactivity of old workers and their low rate of unemployment.

We also perform a second series of exercises in order to decompose the variability of unemploy-

\footnotetext{
${ }^{1}$ In terms of methodology, we follow a rich literature interested in the cyclical behavior of worker flows. See for example Abowd and Zellner (1985), Darby, Haltiwanger, and Plant (1986), Davis (1987), Blanchard and Diamond (1990), Hall (2006), Shimer (2007), Fujita and Ramey (2009), and Elsby, Michaels, and Solon (2009)
} 
ment and participation within age groups. Our results suggest that those transition probabilities which play an important role in the case of differences between age groups are also important when considering the heterogeneity within age groups. The partial exception to this is the job finding rate, which seems important to account for within-age variability but not much for variability between ages.

In light of these facts, we argue that models trying to explain unemployment over the life-cycle should not ignore ins and outs of inactivity. Although labor market models that consider only employment and unemployment can achieve good fit of unemployment stocks over the life-cycle, they are not useful tools to perform counterfactual experiments nor policy analysis. On the other hand, our analysis on sources of variation shows that neglecting inactivity related transitions would left unaccounted at least $40 \%$, and up to $80 \%$ of the variability of unemployment within age.

A growing literature attempts to explain the aggregate levels of unemployment and participation by focusing on search models that specifically include a life-cycle structure. ${ }^{2}$ Those papers are motivated by labor market heterogeneity observed over the life-cycle, which potentially offers tools to explain labor market outcomes at the aggregate level. ${ }^{3}$ Some papers in the literature highlight the importance of ageing, which impacts participation and unemployment rates of older workers. For instance, Hairault, Langot, and Sopraseuth (2010) study the effect of exogenous variation in the retirement age on the employment rate of older workers. Finite horizon induces older workers to leave the labor force because they have fewer incentives to search when unemployed if they anticipate to be employed during a shorter period of time. Ljungqvist and Sargent (2008) offer a quantitative theory of the US-Europe unemployment gap in which the negative effect of labor market institutions have on incentives to search is amplified through faster human capital depreciation among older workers. ${ }^{4}$ Other authors focus on the decrease in the job finding and separation probabilities that mainly occur at the beginning of the life-cycle, which we document in this paper. Menzio, Telyukova, and Visschers (2010) assess the importance of search frictions to generate the

\footnotetext{
${ }^{2}$ The approach in most of these papers is positive, with the exception of Chéron, Hairault, and Langot (2011) and Michelacci and Ruffo (2010), who respectively analyze optimal employment protection and unemployment insurance over the life-cycle.

${ }^{3}$ Aggregate unemployment rates for OECD countries exhibit marked heterogeneity (see for example, table 3 in Jaimovich and Siu (2009)) which is driven mainly by the fate of the young and the old, but not the "prime age" workers. Evidence of this can be found in OECD (1996) for the young and in OECD (1998) for older workers. This sheds light on the importance of taking into account life-cycle considerations and the age composition of the workforce in order to understand aggregate unemployment and participation rates.

${ }^{4}$ See also Kitao, Ljunqvist, and Sargent (2008).
} 
observed life-cycle wage profiles, as opposed to human capital accumulation. Their quantitative exercise requires a precise calibration of transitions between employment and non employment as well as job-to-job mobility. Chéron, Hairault, and Langot (2008) generate a decreasing profile for the job finding and separation probabilities because of finite horizon as in Hairault, Langot, and Sopraseuth (2010). Moreover, their model produces lower employment rates among the older population.

We are motivated by the potential gains life-cycle search models provide. Studying the evolution of worker flows helps identify theories for the behavior of stocks. Accordingly, our results offer some guidelines for the aforementioned literature. The empirical labor market transition probabilities that we provide can be used to assess the ability of quantitative life-cycle simulation models to match the data. It seems that current models can be improved along two dimensions. First, though inactivity is considered in some of them, modeling the decision to return to activity appears as a promising avenue for future research. The literature has carefully studied the transition from activity to inactivity: our results suggest that mechanisms based on the transition back to activity are even more relevant, especially to explain why the unemployment rate does not increase at the end of the life-cycle. Second, paying attention at the mechanisms behind the decrease in the probability of job separation among the population aged below $30^{5}$ is also important.

Further, our results may also be useful from a policy perspective: they give a hint on which transitions a government needs to act in order to influence the level of unemployment and participation of specific age groups. Implementing policies that affect unemployment and participation through these transition probabilities may be more effective than considering policies that operate through others. ${ }^{6}$

The need for inactivity as a third labor market state goes beyond the life-cycle literature. Recently, several authors have pushed this idea in the case of search models. An example in the context of the European unemployment gap is Rogerson (2008). He emphasizes that the decline in employment occurs at a steady pace from 1956 until the mid 1990s, while the increase in unemployment occurs in the mid 1970s. This suggests that theories focusing on the European

\footnotetext{
${ }^{5}$ As it is done for instance in Menzio, Telyukova, and Visschers (2010) and Hairault, Langot, and Sopraseuth (2010).

${ }^{6}$ Of course, the identification of appropriate policies becomes complete once an appropriate policy analysis is implemented. This requires analyzing the impact of a specific policy in hand through a structural model or an experiment. Thinking about welfare consequences is also important. Nevertheless, it is worth mentioning this scope of our results.
} 
labor market dilemma should focus on employment rather than unemployment to understand the opposite evolution of European and US labor markets. We see our results as complementary to this other strand of literature. ${ }^{7}$

The structure of the paper is as follows. In Section 2 we discuss our empirical strategy, including data sources and estimation procedures. Section 3 describes how we construct the age-dependent Markov transition matrices and how we simulate rates of unemployment and participation for a given cohort. In Section 4, we present our main results, while Section 5 asks what are the consequences of ignoring inactivity and Section 6 relates our methodology to existing methods. Finally, Section 7 concludes.

\section{Data and Empirical Analysis}

Our main data source are the basic monthly data files from the Current Population Survey (CPS). ${ }^{8}$ Our sample consists individuals observed between January 1976 and July 2010, inclusively. Throughout the paper, we perform analysis separating male and female samples. In each month (period $t$ ) we identify workers according to their labor force status: employment $\left(e_{t}\right)$, unemployment $\left(u_{t}\right)$ and inactivity/out of the labor force $\left(o_{t}\right)$. Following Shimer (2007), we match individuals across consecutive months based on interview identification numbers, gender, race and age. ${ }^{9}$ This limited longitudinal aspect of the data ${ }^{10}$ is enough to calculate flows between these three employment states for each month. ${ }^{11}$ We define the set of indicator variables $D_{n t}^{x z}$ that take the value of 1 if individual $n$ has transitioned from labor status $x \in\{e, u, o\}$ in period $t-1$ to labor status $z \in\{e, u, o\}$ in $t$ (for expositional reasons, we ignore gender indicators). Then, we take weighted averages of these indicator variables for each month $t$, for each age $a$, and for each birth cohort $c$ to obtain a measure of monthly, age and cohort specific transition probabilities between employment, unemployment

\footnotetext{
${ }^{7}$ Several papers have attempted to extend basic search models to a context with endogenous labor market participation. Examples are Alvarez and Veracierto (1999), Garibaldi and Wasmer (2005), Ravn (2006), Krussel, Mukoyama, Rogerson, and Şahin (2008), Veracierto (2008), Pries and Rogerson (2009) and Krussel, Mukoyama, Rogerson, and Şahin (2010), among others.

${ }^{8}$ Available from the National Bureau of Economic Research, at http://www.nber.org/data/cps_basic.html

${ }^{9}$ The unit of analysis in the CPS is a physical address, hence, the same identification number during two consecutive months might not correspond to the same person. Admittedly, the estimates we provide may be slightly biased since the relatively small sample of movers are qualitatively different from stayers. Other papers using this dataset have the same shortcoming and we comment on this below.

${ }^{10}$ The matching of individuals can only be done for a maximum of 3 consecutive months, given the rotating panel aspect of the CPS.

${ }^{11}$ We built upon Robert Shimer's Stata codes, which are publicly available at http://sites.google.com/site/robertshimer/research/flows
} 
and out of labor force states. We denote $\mathbb{I}(a, t, c)$ as an indicator variable that takes the value of 1 if the individual is observed in month $t$, belongs to cohort $c$ and is $a$ years old, and 0 otherwise. Then, we define the corresponding worker flow $f_{\text {atc }}^{x z}$ as follows:

$$
f_{\text {atc }}^{x z}=\frac{\sum_{n=1}^{N} D_{n t}^{x z} \cdot \omega_{n t} \mathbb{I}(a, t, c)}{\sum_{n=1}^{N} D_{n t}^{x} \cdot \omega_{n t} \mathbb{I}(a, t, c)}
$$

where $D_{n t}^{x}$ equals 1 if the individual was in state $x$ in $t-1$ and $\omega_{n t}$ is the sample weight.

Our goal is to extract life-cycle profiles for each transition. Since age-time-cohort bins are constructed from samples of different size, we weight each transition by the square root of the sample size of the original state (which we denote by $N_{\text {atc }}^{x}$ ) during estimation. In this way, the variance of the estimated error does not depend on the sample size of the bin. Hence, our typical regression equation is ${ }^{12}$

$$
f_{a t c}^{x z} \sqrt{N_{a t c}^{x}}=\sum_{a=1}^{A} \gamma_{a}^{x z} D_{a t c} \sqrt{N_{a t c}^{x}}+\beta W_{a t c} \sqrt{N_{a t c}^{x}}+\epsilon_{a t c} \sqrt{N_{a t c}^{x}}
$$

where $\left\{D_{a t c}\right\}$ are age dummy variables and $\left\{\gamma_{a}^{x z}\right\}$ are their corresponding estimated coefficients, our statistics of interest. $W_{a t c}$ is a vector of control variables. In what follows, we show results for unconditional age-specific transition probabilities, because the lessons we obtain from the data remain roughly unchanged after controlling for several standard observable variables. In the Appendix we perform a series of robustness analysis of our results by computing transition probabilities conditional on cohort, time, and state effects. ${ }^{13}$ The quantitative results of unconditional and conditional estimates are virtually identical.

To allow for a more efficient estimation, we implement a Seemingly Unrelated Regressions (SUR) method, where we estimate jointly the age profiles for each transition probability, including equations for unemployment and participation rates. If we had a set of standard unweighted equations with identical regressors, there would be no benefit for a pooling procedure: equationby-equation OLS would be enough to achieve efficiency. However, sample-size weighting essentially

\footnotetext{
${ }^{12}$ In our subsequent analysis, we do not need estimates of staying probability transitions $e e, u u, o o$, so we discard them from the presentation.

${ }^{13}$ Since we obtain our age-specific estimates from individuals of the same age at different time periods, it makes sense to control for cohort effects. Similarly, we control for time and US state effects because there are significant fluctuations in labor market transition probabilities at business cycle frequencies and across states.
} 
introduced different regressors in each equation, making the SUR method a more efficient choice. Moreover, we want to uncover the whole joint stochastic profile of labor market transitions along the life-cycle. This multidimensional profile is key to study how important the volatility of each specific flow is in accounting for the volatility of unemployment and participation along the life-cycle. We denote by $\Omega$ the variance-covariance matrix of the estimated $\left\{\gamma_{a}^{x z}\right\}$.

We depict the unconditional estimated life-cycle profiles (the collection of $\left\{\gamma_{a}^{x z}\right\}$ ) in Figures 1 and 2 below in the case of the male and female population respectively. ${ }^{14}$ The shaded areas around the profiles are $95 \%$ confidence bounds constructed from the estimated standard deviations of the SUR model.

Figure 1: Life-cycle profiles of flows between employment $(e)$, unemployment $(u)$ and out of the labor force (o) (Males)
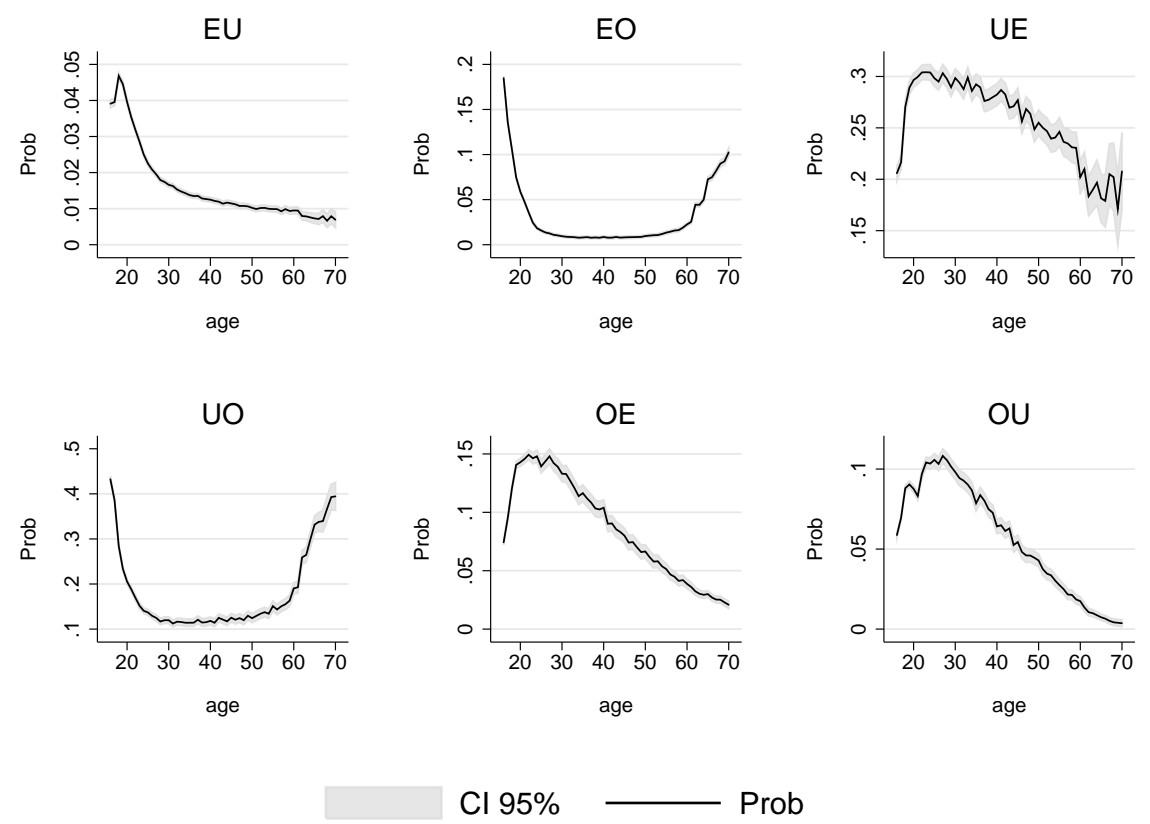

Note: Unconditional life-cycle profiles estimated via Seemingly Unrelated Regressions (SUR).

In Figures 1 and 2 we show the transition probabilities for both male and female workers between the ages of 16 and 70. Besides level differences, the male and female profiles are quite similar. Qualitatively, for both genders the employment-to-unemployment $(E U)$, employmentto-inactivity $(E O)$ and the unemployment-to-inactivity $(U O)$ transition probabilities have stable

\footnotetext{
${ }^{14}$ Since we are interested in the average transition probability conditional on $W_{\text {atc }}$, our linear regression model does not have an intercept.
} 
Figure 2: Life-cycle profiles of flows between employment $(e)$, unemployment $(u)$ and out of the labor force $(o)$ (Females)
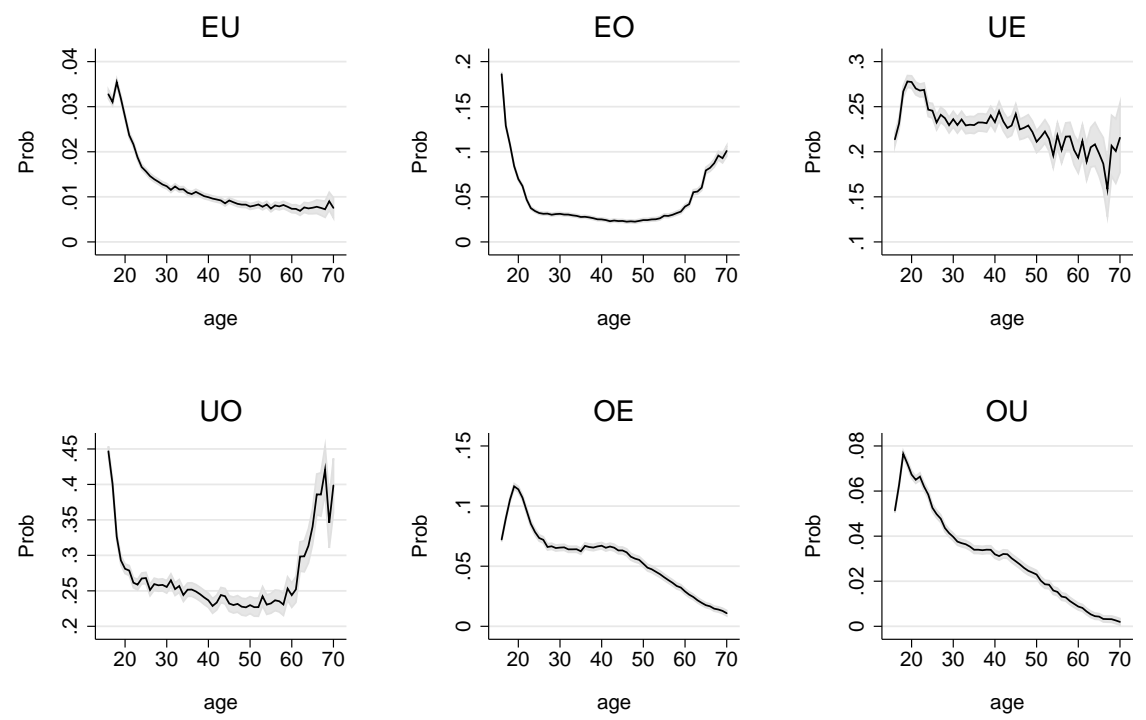

$\mathrm{Cl} 95 \%-$ Prob

Note: Unconditional life-cycle profiles estimated via Seemingly Unrelated Regressions (SUR).

patterns between 30 to 60 years of age, while they show a negative slope at younger ages and an increase for older workers. The job finding probability $(U E)$ shows an increase until the mid20's and then a slight but persistent decrease. The probabilities of going from inactivity to both employment and unemployment $(O E$ and $O U)$ show a hump shaped pattern, peaking around age 30 and steadily decreasing from that point to age 70 . Confidence bands show that the estimated age profiles are precise, with the exception of $U E$.

As for quantitative (beside level) differences, the female sample exhibits a flatter profile of job finding probabilities and less pronounced hump shape patterns of $O E$ and $O U$. The main differences are concentrated around ages 20 to 30, so they are probably linked to fertility and child rearing.

\section{Markov Chain Analysis}

In this section, we propose a way to account for the contribution of each transition probability into the determination of participation $\left(P_{a}=1-o_{a}\right)$ and unemployment $\left(U_{a}=u_{a} /\left(e_{a}+u_{a}\right)\right)$ profiles over the life-cycle. Once we get our estimates for transition probabilities, we construct age-specific Markov transition matrices denoted $\Gamma_{a}$. Starting from initial conditions of labor force status at 
some starting age $S_{1}$, we compute the predicted labor market states after twelve months as

$$
S_{2}=\Gamma_{1}^{12} S_{1} \quad \text { with } \Gamma_{1}=\left(\begin{array}{ccc}
\gamma_{1}^{E E} & \gamma_{1}^{E U} & \gamma_{1}^{E O} \\
\gamma_{1}^{U E} & \gamma_{1}^{U U} & \gamma_{1}^{U O} \\
\gamma_{1}^{O E} & \gamma_{1}^{O U} & \gamma_{1}^{O O}
\end{array}\right) \text { and } S_{1}=\left(\begin{array}{c}
e_{1} \\
u_{1} \\
o_{1}
\end{array}\right)
$$

Using the same logic, we can obtain the probability of labor market states at any age $a$ by doing the following calculation

$$
S_{a}=\left(\prod_{i=1}^{a-1} \Gamma_{i}^{12}\right) S_{1}
$$

It is important for the exercise to distinguish between annual and monthly transition probabilities. Figures 3 and 4 show the differences between the two time horizons. In the Figures, the dotted lines are the implied transitions when we iterate the age transition matrices twelve times and compute the specific probabilities, while the solid lines are the monthly transitions (already depicted in Figures 1 and 2).

Figure 3: Monthly vs Annual transition probabilities (males)
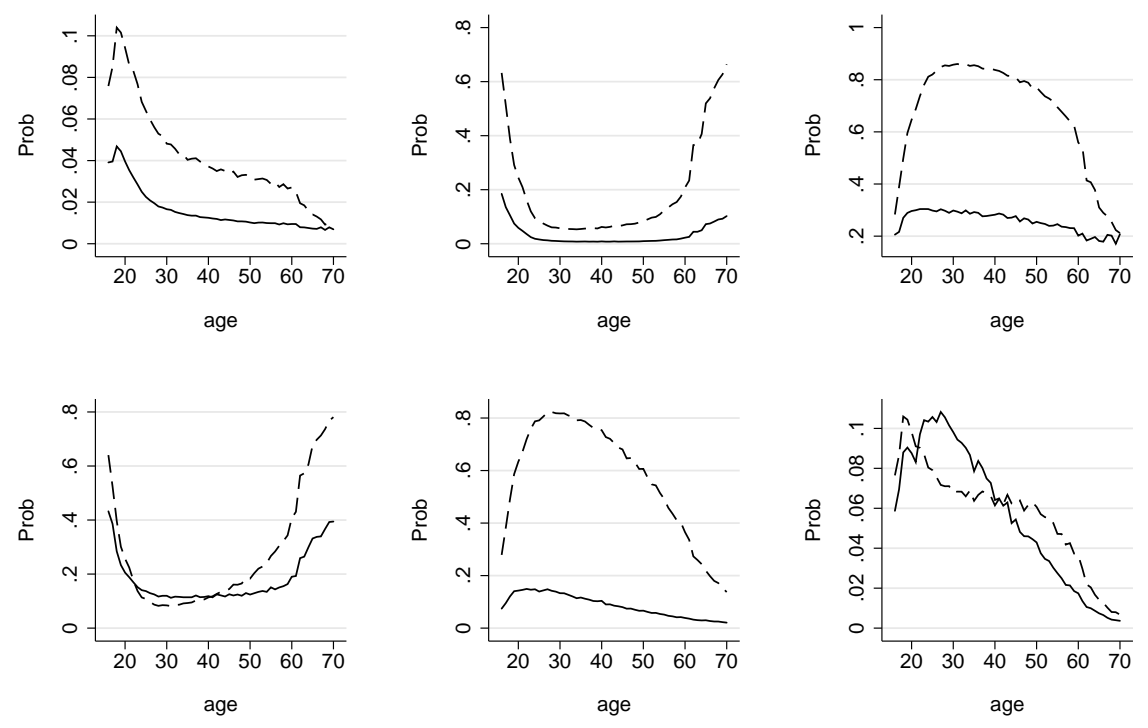

monthly

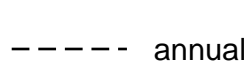

Note: Unconditional life-cycle profiles estimated via Seemingly Unrelated Regressions (SUR).

Using equation (2), we can obtain complete lifetime profiles implied by the estimated transition 
Figure 4: Monthly vs Annual transition probabilities (females)
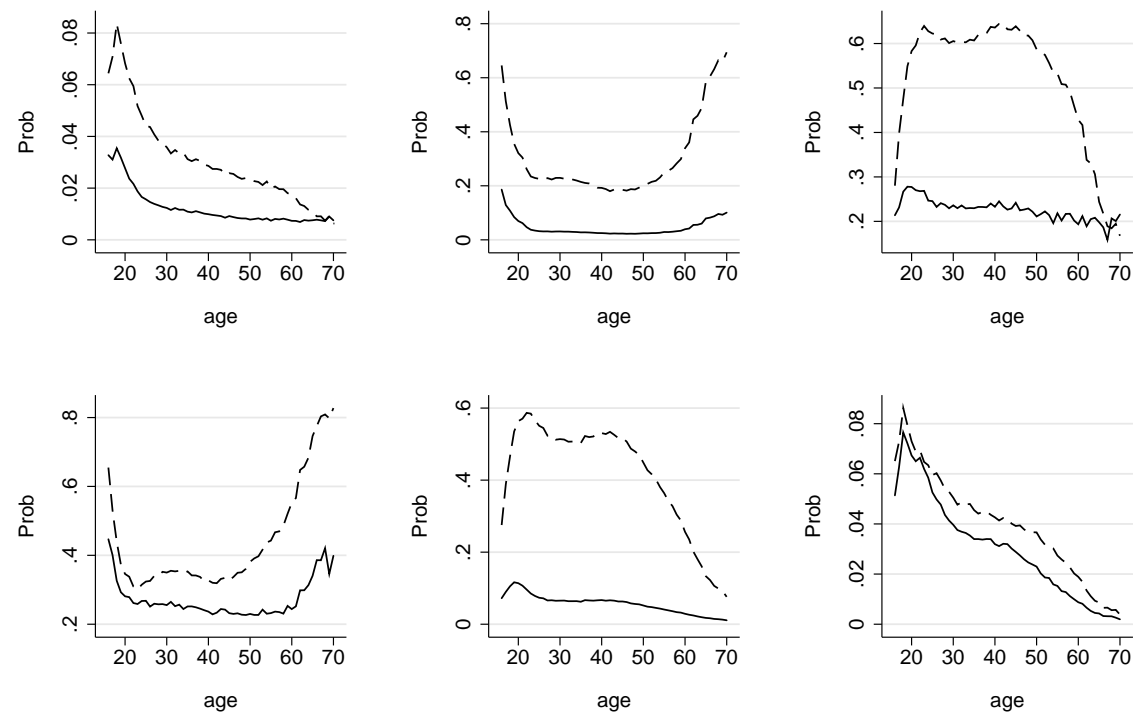

monthly

annual

Note: Unconditional life-cycle profiles estimated via Seemingly Unrelated Regressions (SUR).

probabilities, i.e., $U_{a}=u_{a} /\left(e_{a}+u_{a}\right)$ and $P_{a}=1-o_{a}$ using observed initial conditions. We compare the computed lifetime sequences of participation and unemployment to the actual lifetime profiles obtained from the data. The results are depicted in Figure 5. The estimated transition probabilities come remarkably close to replicate the actual profiles. In each subfigure we also show the value of $1-R^{2}$, where $R^{2}$ is the $\mathrm{R}$-squared of a linear regression between the actual profile and the counterfactual.

Looking at the female sample, participation exhibits a hump around ages 25 to 40, which can be linked to reduced participation due to fertility and child rearing. Our method replicates this pattern, given the gender specific estimates of $O E$ and $O U$ : these transition probabilities are lower for females than for males during these years, representing women's choices not to rejoin the labor force if not participating. 
Figure 5: Unemployment and Participation according to annual probabilities
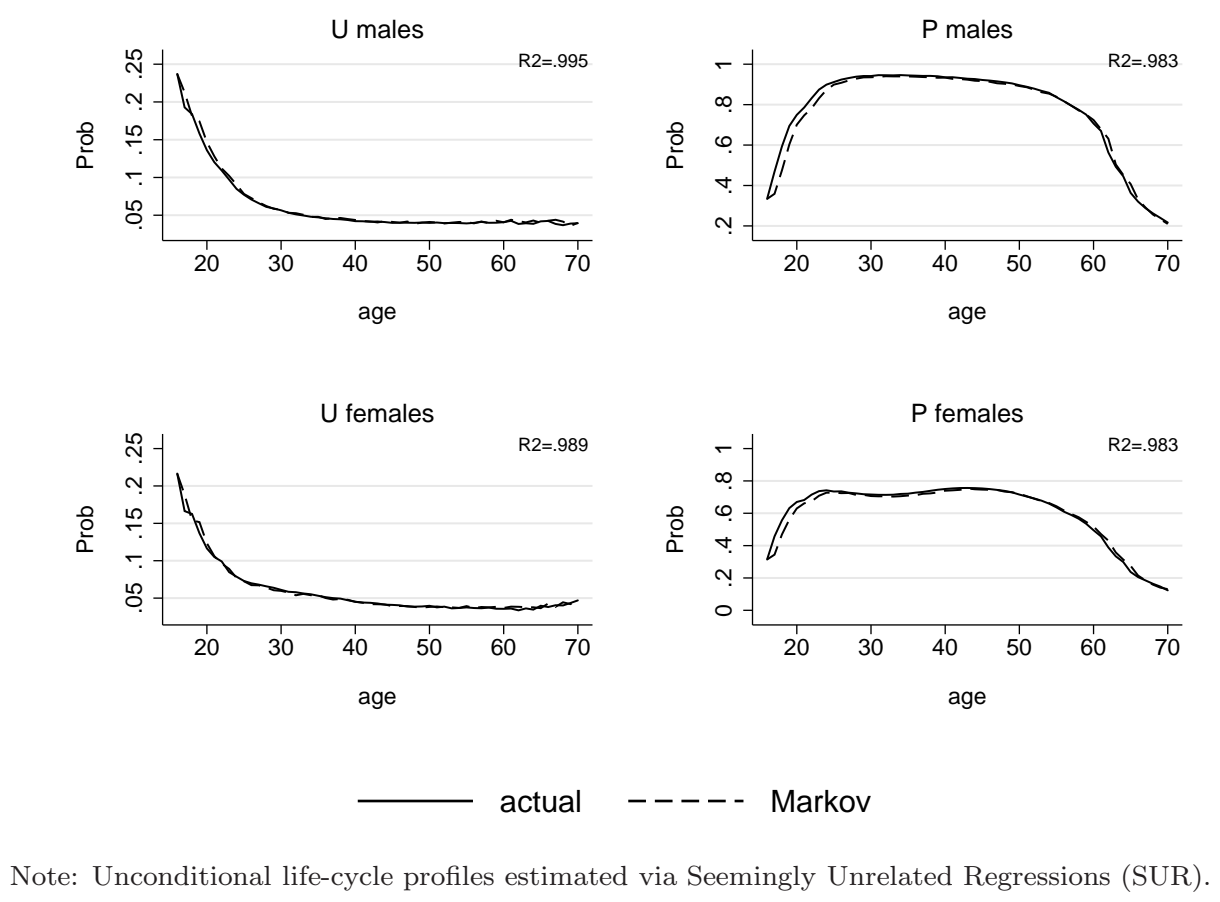

\section{Results}

\subsection{Average Profiles}

With the constructed transition matrices, we perform a set of decomposition exercises. We want to understand how influential is each specific flow on its own for the determination of unemployment and participation profiles. Thus, we fix the age-specific transition probability of each flow (EU, $E O, U E, U O, O E, O U)$ at an arbitrary age. Then, we adjust the probability of "staying" flows $(E E, U U, O O)$ so that the monthly transition matrices are well defined. ${ }^{15}$ The remaining five independent transition probabilities are left unchanged. Using this alternative set of age-specific transition matrices, we can assess the contribution of a specific flow by inspecting the loss of goodness-of-fit derived from such a change. We call this method "all but one change" (AB1C). We interpret this procedure as an approximated model-free counterfactual profile of unemployment and participation, the closest thing to an "all else constant" exercise to assess the effect of a particular flow on the level of the stock. This method has the advantage of introducing minimal changes

\footnotetext{
${ }^{15}$ Suppose we fix $\gamma_{a}^{E U}=\bar{\gamma}^{E U}$ for all $a$. We then adjust the transition matrices for all ages by computing $\widetilde{\gamma}_{a}^{E E}=$ $1-\bar{\gamma}^{E U}-\gamma_{a}^{E O}$.
} 
to what an underlying structural general equilibrium model would provide for these transition probabilities.

Figures 6 to 9 depict the alternative unemployment and participation profiles for both male and female workers when the particular transition probabilities related to each subfigure are replaced by their life-cycle averages. For example, the first subfigure in Figure 6 shows how different life-cycle unemployment rates would be if the separation rate $(E U)$ were the same across all ages (fixed at the life-cycle average), instead of being age-specific. Hence, whenever there is a significant difference between both lines, we argue that the particular transition probability contributes to the shape of the life-cycle profile in either participation or unemployment rates. ${ }^{16}$

Figure 6: Importance of flows in Markov chains (AB1C): Unemployment, males
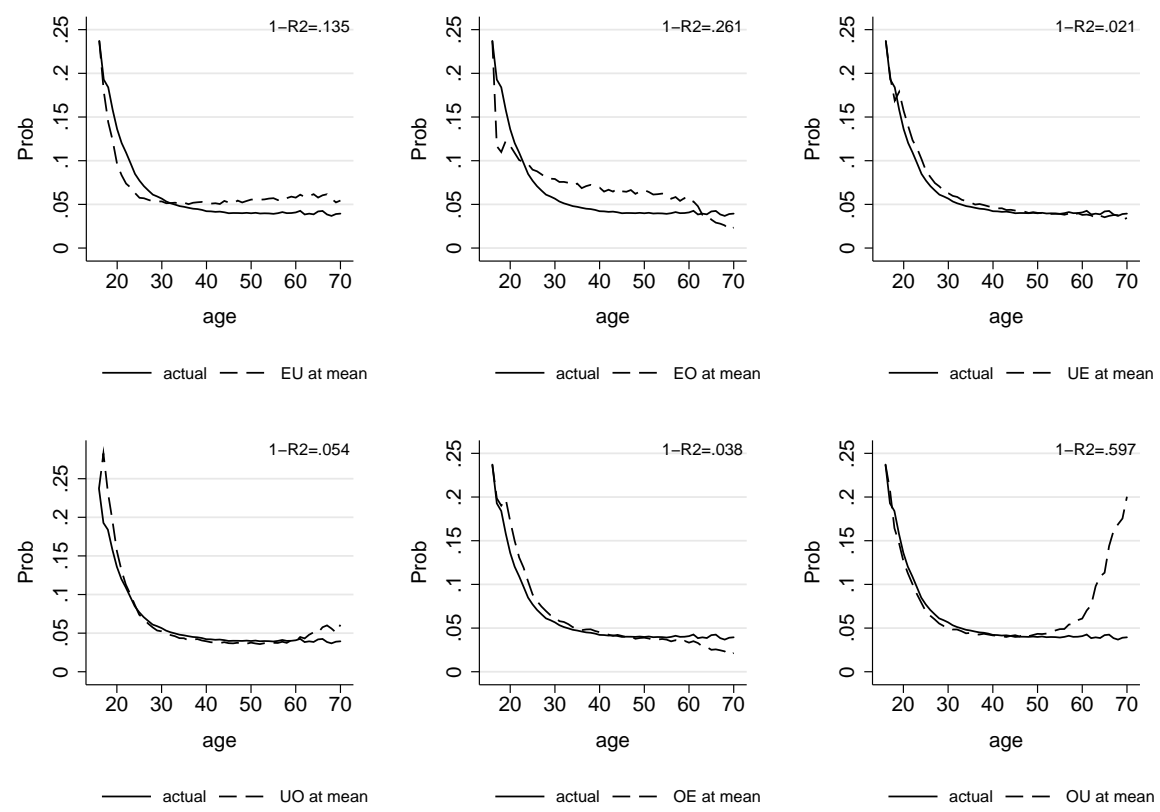

Note: Unconditional life-cycle profiles estimated via Seemingly Unrelated Regressions (SUR).

Looking at Figures 6 and 7 we observe that implied unemployment profiles are barely affected

\footnotetext{
${ }^{16}$ We also perform an alternative exercise. We assess the specific contribution of only one transition rate by constructing Markov transition matrices that keep fixed all but one transition probabilities at the average life-cycle level. We label this method as the "all but one fixed" (AB1F) decomposition. The AB1F method need five out of six transitions to remain fixed, which presumably would imply severe general equilibrium effects in an underlying structural model. Accordingly, we strongly prefer the AB1C method, since it resembles more closely a counterfactual analysis, departing only marginally from estimated values. Nevertheless, results from the AB1F decomposition are quite similar to those obtained from the preferred method. They are available upon request.
} 
Figure 7: Importance of flows in Markov chains (AB1C): Unemployment, females
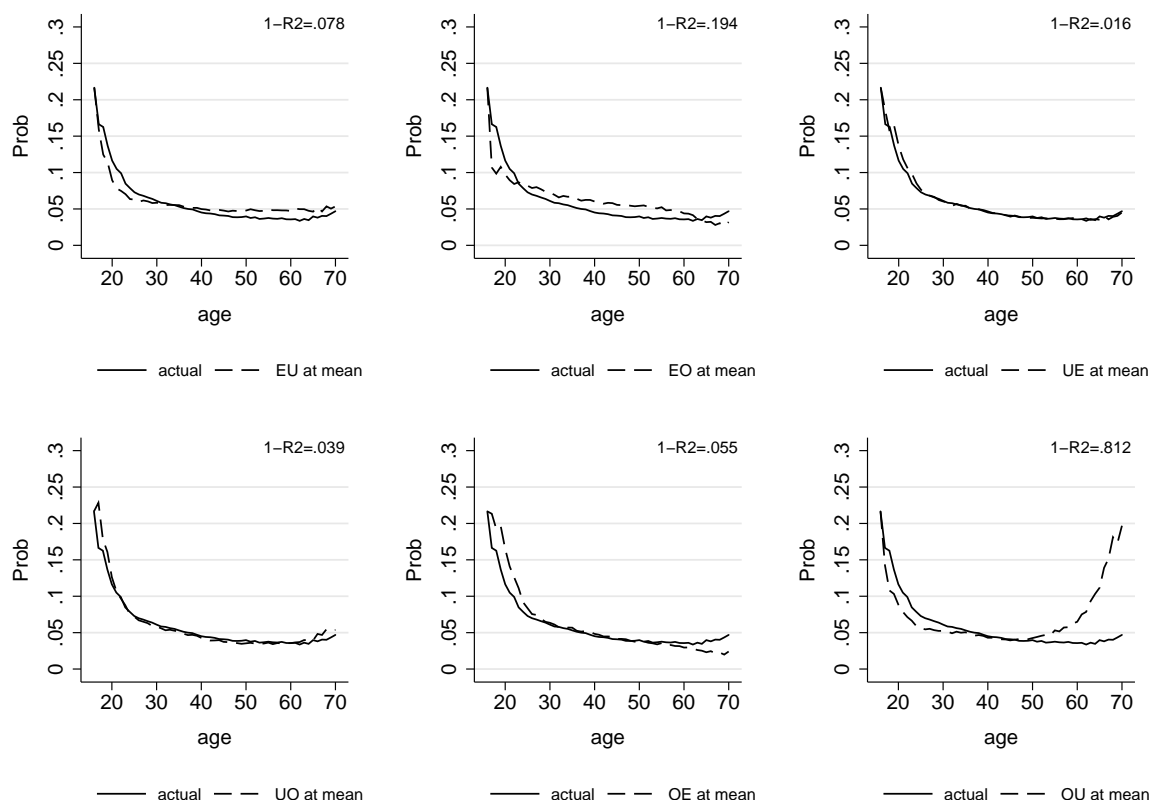

Note: Unconditional life-cycle profiles estimated via Seemingly Unrelated Regressions (SUR).

by changes in the job finding $(U E)$ and the inactivity-to-employment transition $(O E)$ probabilities. Changes in $E U$ (separation probability) matter to explain higher unemployment of the young and lower unemployment of the old. A larger effect occurs with changes in "retirement" probabilities. If the $E O$ flows were at its average level, we would observe smaller young unemployment and higher old unemployment rates. Finally, the probability of transiting from inactivity to unemployment plays a role in explaining low unemployment rates for workers older than 55 years of age. This decomposition exercise shows that the decision of searching for a job once workers have been inactive is the most important factor explaining low unemployment rates for old workers. Again, besides a level effect, results are similar for both male and female population groups and the qualitative effects of specific transition probabilities on unemployment rates seem to work identically across genders.

Figures 8 and 9 show the decomposition for the participation rate. The pictures show that neither separation $(E U)$, unemployment-to-inactivity $(U O)$ nor job finding probabilities $(U E)$ explain much of the age differences in participation rates. If these probabilities were fixed at their average 
Figure 8: Importance of flows in Markov chains (AB1C): Participation, males
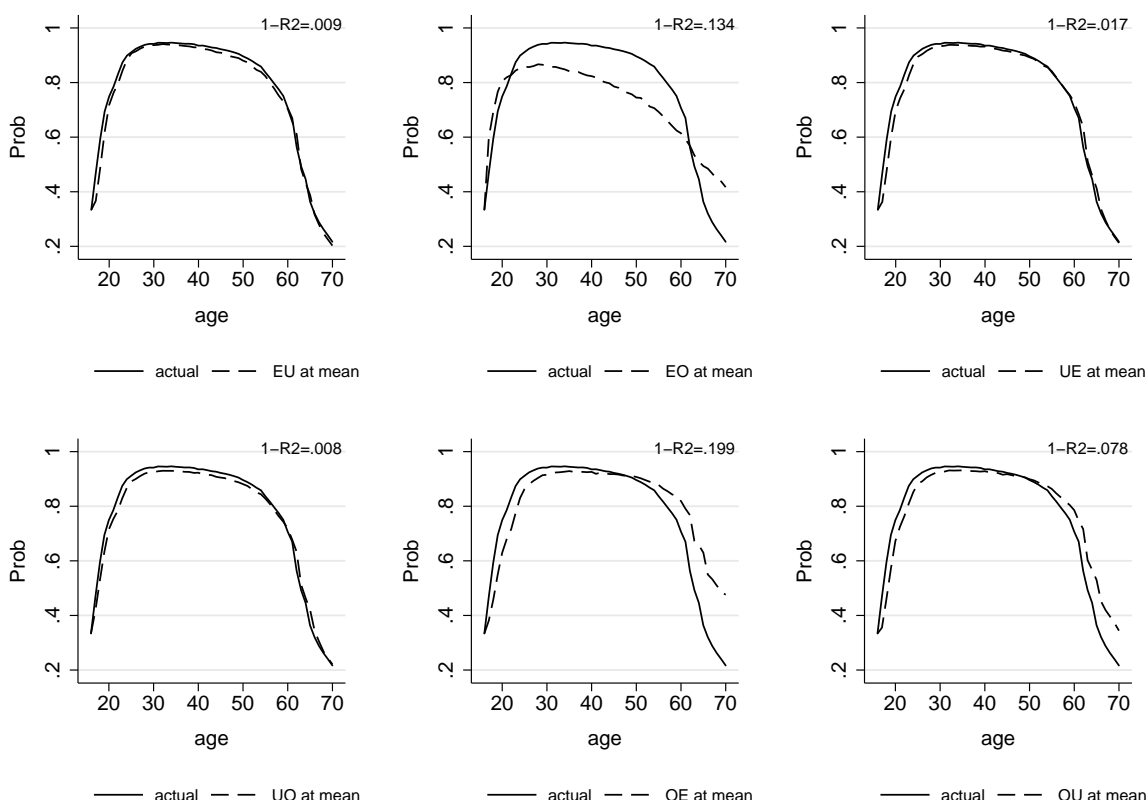

Note: Unconditional life-cycle profiles estimated via Seemingly Unrelated Regressions (SUR).

life-cycle levels, we would observe a somehow lower participation for workers older than 40 . The most important life-cycle changes that influence participation profiles come from the "retirement" probability $(E O)$. The evolution of this transition probability over the cycle increases labor force participation by as much as $20 \%$ at age 60 . Finally, the $O E$ flow seems to be quite important to explain higher participation for the young and, especially, lower participation for the old.

Admittedly, the choice of keeping each transition probability fixed at its average life-cycle value is arbitrary. We could also focus on another type of question. For example, what would be the unemployment or participation life-cycle profiles if all workers had the same job finding probability of a 20 year old worker ( $U E$ transition)? We can answer this question by using the AB1C decomposition with fixed probabilities at arbitrary ages. Below we focus on young (20), prime age (40) and old (60) workers.

Table 1 summarizes the results from the decomposition exercise as well as the ones of the baseline (average probability) case. This table quantifies the qualitative examinations we perform on Figures 6-9. Here we assess the explanatory power for the AB1C method as the drop in goodnessof-fit generated by keeping constant a particular probability of transition. Hence, our measure of 
Figure 9: Importance of flows in Markov chains (AB1C): Participation, females
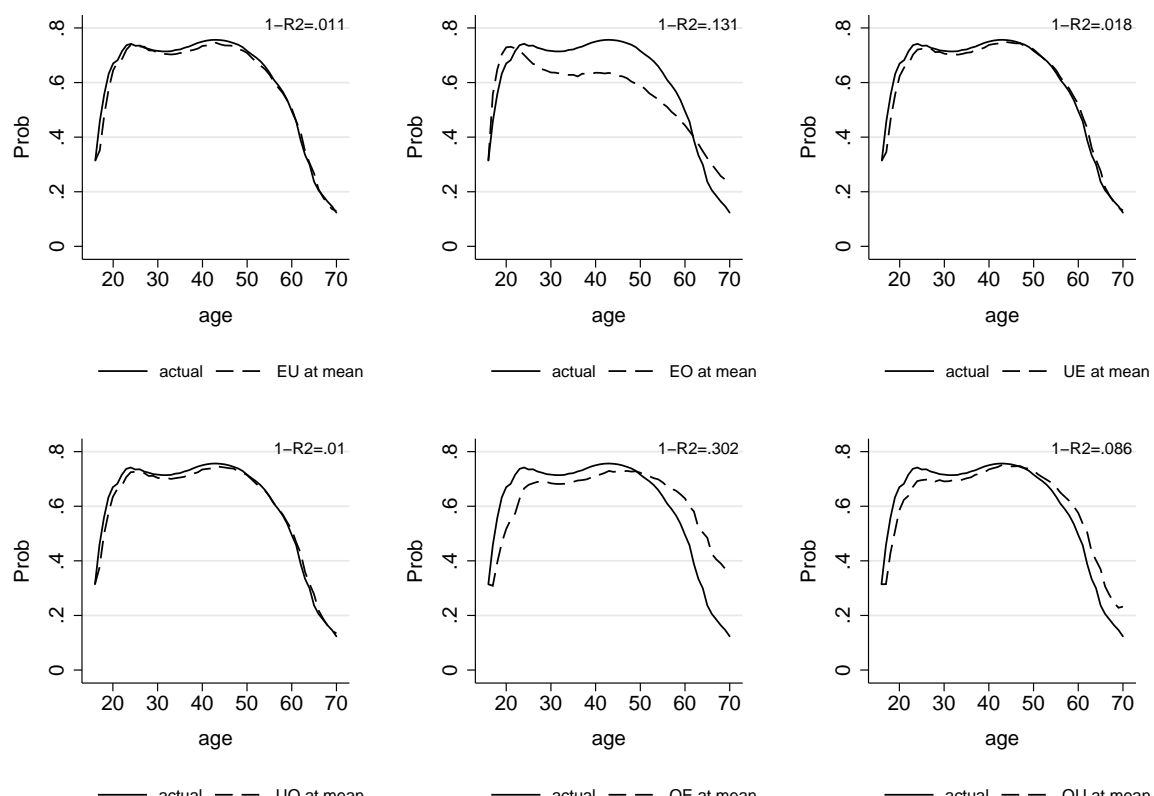

Note: Unconditional life-cycle profiles estimated via Seemingly Unrelated Regressions (SUR).

explanatory power in this case is $1-R_{x z}^{2}$, where the last term is the $\mathrm{R}$-squared from a regression between the actual life-cycle profile of unemployment/participation and the one simulated via an adjusted Markov chain with the flow $x z$ fixed at the specified age.

From Table 1 we see that the $E O$ and $O U$ flows are the most influential ones in determining the life-cycle trajectories of unemployment. For instance, if a worker keeps his $E O$ transition probability fixed at its 20-year level, the Markov chain analysis would explain $85 \%$ less than what it could if we allowed age dependent $E O$ values. Once we fix the $E O$ probability at other ages, this number decreases to $20 \%$. Thus, the $E O$ flow is particularly important to determine unemployment for young male workers. In contrast, the $O U$ flow plays an important role in shaping unemployment at all ages.

The $O E$ flow plays a small role in shaping unemployment, but is quite relevant for participation. We can interpret this latter flow as the job finding probability of workers exerting little effort to find a job. These transitions are particularly important for young and old workers. An interesting observation is the lack of relevance of the job finding probability ( $U E$ flow) at any stage in the 
Table 1: Unemployment and Participation Flow Decompositions

\begin{tabular}{|c|c|c|c|c|c|c|c|}
\hline \multicolumn{8}{|c|}{ Males } \\
\hline Fixed probs & & $E U$ & $E O$ & $U E$ & $U O$ & $O E$ & $O U$ \\
\hline \multirow[t]{2}{*}{$\gamma^{x z}$ fixed at 20} & $\bar{U}$ & 18.5 & 39.2 & 1.4 & 4.8 & 4.6 & 64.8 \\
\hline & $P$ & 0.1 & 19.4 & 1.7 & 0.9 & 28.2 & 10.3 \\
\hline \multirow[t]{2}{*}{$\gamma^{x z}$ fixed at 40} & $U$ & 13.3 & 18.1 & 1.6 & 7.7 & 3.8 & 61.6 \\
\hline & $P$ & 0.1 & 23.0 & 1.7 & 0.7 & 22.7 & 8.5 \\
\hline \multirow[t]{2}{*}{$\gamma^{x z}$ fixed at 60} & $U$ & 13.3 & 22.4 & 4.2 & 5.2 & 6.4 & 33.9 \\
\hline & $P$ & 1.1 & 12.4 & 1.7 & 0.9 & 11.8 & 4.9 \\
\hline \multirow[t]{2}{*}{$\gamma^{x z}$ fixed at avg } & $U$ & 13.5 & 26.1 & 2.1 & 5.4 & 3.8 & 59.7 \\
\hline & $P$ & 0.9 & 13.4 & 1.7 & 0.8 & 18.6 & 7.8 \\
\hline \multicolumn{8}{|c|}{ Females } \\
\hline & & $E U$ & $E O$ & $U E$ & $U O$ & $O E$ & $O U$ \\
\hline \multirow[t]{2}{*}{$\gamma^{x z}$ fixed at 20} & $U$ & 7.7 & 25.2 & 1.1 & 3.7 & 2.7 & 86.6 \\
\hline & $P$ & 0.8 & 18.7 & 1.8 & 0.1 & 45.8 & 14.5 \\
\hline \multirow[t]{2}{*}{$\gamma^{x z}$ fixed at 40} & $U$ & 7.9 & 16.9 & 1.5 & 4.7 & 4.3 & 81.8 \\
\hline & $P$ & 1.2 & 9.5 & 1.8 & 0.9 & 34.0 & 8.9 \\
\hline \multirow[t]{2}{*}{$\gamma^{x z}$ fixed at 60} & $U$ & 13.3 & 22.4 & 4.2 & 5.2 & 6.4 & 33.9 \\
\hline & $P$ & 1.3 & 11.7 & 1.8 & 0.9 & 17.0 & 5.5 \\
\hline \multirow[t]{2}{*}{$\gamma^{x z}$ fixed at avg } & $U$ & 7.8 & 19.4 & 1.6 & 3.9 & 5.5 & 81.2 \\
\hline & $P$ & 1.1 & 13.1 & 1.8 & 1.0 & 30.2 & 8.6 \\
\hline
\end{tabular}

NOTES: The numbers represent the loss of goodness of fit measured by $1-R_{x z}^{2}$, where $R^{2}$ is the R-squared from a regression between the actual life-cycle profile of unemployment/participation and the one simulated via an adjusted Markov chain with the flow $x z$ fixed at the specified age.

life-cycle. The latter observation contrasts with the protagonism of this flow in explaining business cycle variation of unemployment, as noted by Hall (2006) and Shimer (2007).

\subsection{Heterogeneity within age groups}

The previous analysis highlights how the differences in stocks between age groups can be explained by differences in transition probabilities. We now develop a methodology to identify which transition probabilities are behind the heterogeneity in unemployment and participation within each age group. Our aim is to assess the contribution of the variance of a particular flow to the variance of unemployment and participation at a given age $a$, which we denote by $\operatorname{Var}\left(U_{a}\right)$ and $\operatorname{Var}\left(P_{a}\right)$.

Conceptually, we take the SUR estimates $\left\{\gamma_{a}^{x z}\right\}$ as a sequence of average transition flows from $x$ to $z$, conditional on age $a$. At the individual level, there is a great deal of variation conditional on age $a$. The procedure we follow attempts to uncover the specific contribution of a flow $x z$ at age $a$ for the variability of $U$ and $P$. To do this, our first step is to draw 10,000 realizations of the stochastic multivariate profile of transition probabilities over the life-cycle from the SUR estimates, assuming joint normality, i.e. the joint profile has a mean vector given by the estimated $\left\{\gamma_{a}^{x z}\right\}$ (for 
all $x z \in\{E U, E O, U E, U O, O E, O U\}$ and all ages $a=1, . ., A)$ and a variance-covariance matrix $\Omega{ }^{17}$ For the second step, we fix initial conditions at age 16 and compute the 10,000 life-cycle profiles of unemployment and participation with each one of the 10,000 simulated profiles by means of the Markov chain method described in the preceding section. Third, once we obtain the 10,000 stochastic profiles of $U$ and $P$, we calculate the corresponding variances of unemployment and participation for each age group across all simulations. Given that the stocks can be obtained very accurately from transition probabilities assuming a first-order Markovian process, the computed variances should closely approximate $\operatorname{Var}\left(U_{a}\right)$ and $\operatorname{Var}\left(P_{a}\right)$, abstracting from sample size considerations. Fourth, we compare those variances with the ones obtained when the stochastic influence of the flow $x z$ is shut down in order to assess the contribution of that particular flow. In the latter case, $x z$ is thus restricted to be the SUR estimated life-cycle profile (i.e. the "average" life-cycle profile of this flow). We denote these additional statistics as $\operatorname{Var}\left(U_{a} \mid \gamma^{x z}\right)$ and $\operatorname{Var}\left(P_{a} \mid \gamma^{x z}\right)$. Finally, we compute the ratios $Q_{a}^{U, x z}=1-\frac{\operatorname{Var}\left(U_{a} \mid \gamma^{x z}\right)}{\operatorname{Var}\left(U_{a}\right)}$ and $Q_{a}^{P, x z}=1-\frac{\operatorname{Var}\left(P_{a} \mid \gamma^{x z}\right)}{\operatorname{Var}\left(P_{a}\right)}$. We call this method "all but one change" (AB1C) for variance.

The ratios $Q_{a}^{U, x z}$ and $Q_{a}^{P, x z}$ give the relative contribution of a given flow $x z$ to the variance of unemployment and participation of age group $a$, a large value indicating a large contribution. The ratios are displayed on Figure 10 in the case of unemployment, while Figure 11 depicts those related to participation. ${ }^{18}$ They suggest that those transition probabilities which play an important role in the case of differences between age groups are also important when considering the heterogeneity within age groups. Moreover, results are again similar for both men and women.

In the case of the variance of unemployment of the young (say up to 30 years old), almost all flows (except the $U O$ transitions) display modest $Q_{a}^{U, x z}$ statistics, with contributions around $20 \%$. Interestingly, the job finding probability is now the flow that contributes the most for these age groups, especially in the case of men younger than 20 years old (with $Q_{a}^{U, U E}$ statistics around $40 \%$ ). The probability of transiting from employment to unemployment greatly influences the variance of unemployment of the prime-aged (say from 30 to 55 years old), with $Q_{a}^{U, E U}$ statistics that can reach almost $60 \%$ for men and $40 \%$ for women. Finally, Figure 10 indicates that the variance among

\footnotetext{
${ }^{17}$ Normality is justified by standard asymptotic normality of SUR estimator. In practice, we draw a sequence of i.i.d. standard normal realizations and transform it into a realization of the profile using a Cholesky decomposition of the variance matrix $\Omega$.

${ }^{18}$ Notice that the ratios may sometimes take negative values. This may happen because of non-zero covariances may generate an increase in variance when a flow is shut down.
} 
Figure 10: Variance decomposition by age group (AB1C): Unemployment
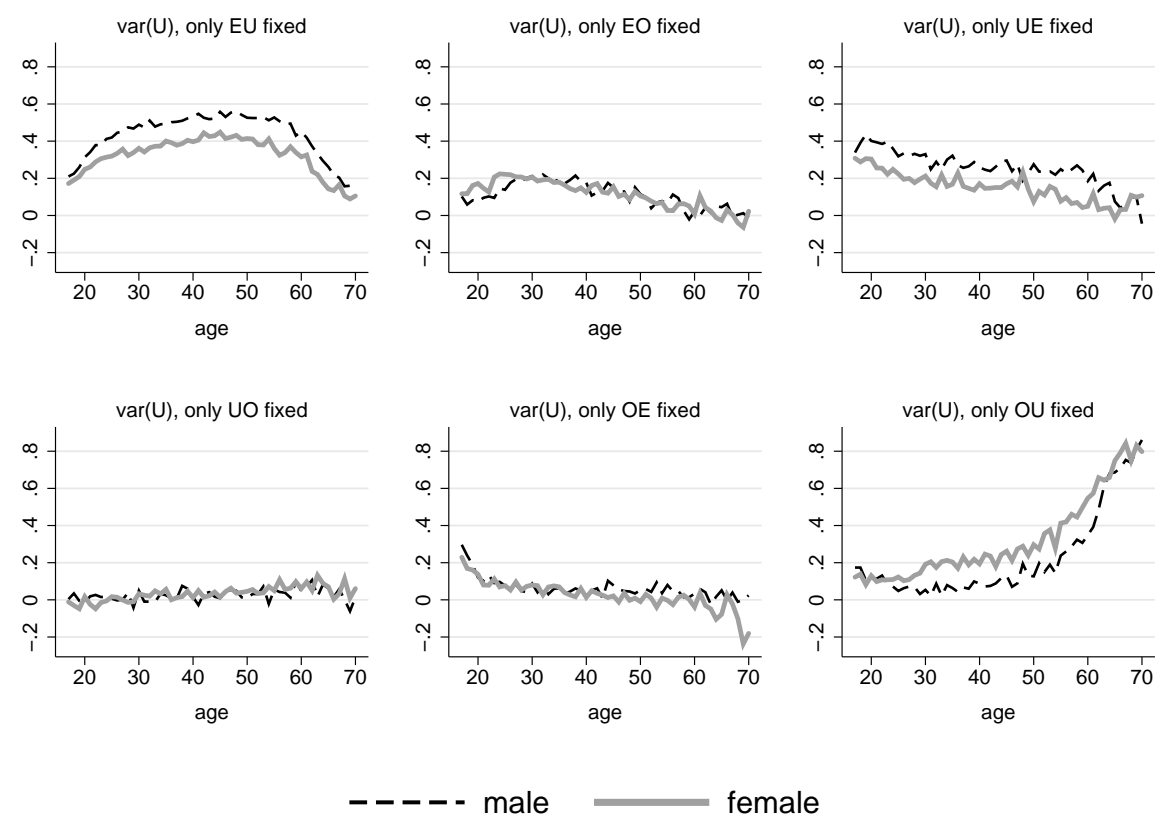

Note: Figures show the percentage of $\operatorname{var}(U)$ at each age explained by fixing one flow at a time at its estimated profile. Based on 10000 simulations, SUR weighted unconditional estimates.

older groups is mainly explained by the variability in the $O U$ transition probability: the respective $Q_{a}^{U, O U}$ statistics steadily increase from about $30 \%$ in the case of the 55 years old population to about $80 \%$ for those aged 70 (for both men and women).

Figure 11 shows the importance of the $E O$ and $O E$ transition probabilities in the case of participation. Each one of these two flows manifests itself at specific moments of the life-cycle and its contribution mirrors the contribution of the other one. The $O E$ transition becomes important in the case of the young and older populations (with $Q_{a}^{P, O E}$ statistics that can respectively reach $60 \%$ and $80 \%$ ), while the $E O$ transition tends to influence the variance of the prime-aged (with $Q_{a}^{P, E O}$ statistics that can reach $80 \%$ in the case of males and $60 \%$ for women). Notice the differences between males and females over the prime-age periods: the contribution of the $O E$ flow is 20 percentage points larger in the case of females, while this difference is compensated by a larger $E O$ contribution in the case of males. Finally, the $O U$ transition is somewhat important in the case of the young and older populations (around 20\%).

The exercise on the variance within age group is subject to the following caveat. The estimated 
Figure 11: Variance decomposition by age group (AB1C): Participation
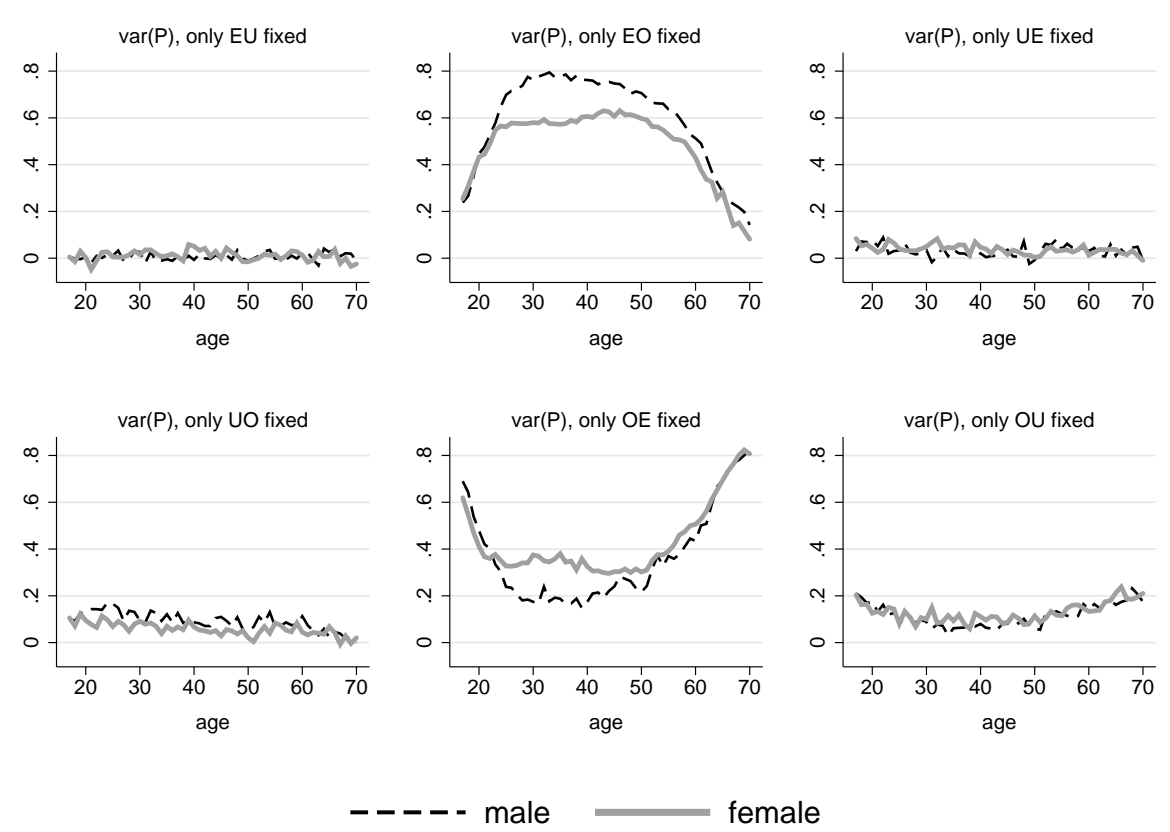

Note: Figures show the percentage of $\operatorname{var}(U)$ at each age explained by fixing one flow at a time at its estimated profile. Based on 10000 simulations, SUR weighted unconditional estimates.

variance-covariance matrix $\Omega$ is influenced by the heterogeneity within group but also by the sample size of the respective $\left\{\gamma_{a}^{x z}\right\}$ estimates. Ideally we would like our results to be the outcome of the former, with the latter playing no role. It turns out that this is not true because the levels of unemployment and participation are not constant over the life-cycle, implying that some transition probabilities are better estimated for some age groups because they are characterized by a larger sample size. For example, the estimated $O E$ and $O U$ transitions are more precise among the older populations because they are characterized by a larger proportion of non-participants, while the precision of the estimates of the $E U, E O, U E$ and $U O$ transition probabilities is worse for these age groups. However, a comparison of the size of the confidence bands in Figures 1 and 2 with the importance of the contributions in Figures 10 and 11 suggests that this caveat is not quantitatively important: it turns out that the flows that display the largest $Q_{a}^{U, x z}$ and $Q_{a}^{P, x z}$ statistics are also estimated with more precision when their contribution is the highest. Hence, their $Q_{a}^{U, x z}$ and $Q_{a}^{P, x z}$ statistics reported in Figures 10 and 11 can be seen as a lower bound. See also the discussion in Section 6.2 . 


\section{Ignoring transitions to and from inactivity}

\subsection{Average Profiles}

What happens if we abstract from inactivity in our models, as a majority of search and matching models do? To answer this question we extend the ideas regarding the AB1C decomposition, but we allow both $U E$ and $E U$ to be fixed at arbitrary values over the life-cycle. We denote this decomposition as "all but two change" (AB2C). Alternatively we could ask how unemployment and participation profiles would look like if we only allow $E U$ and $U E$ transitions to occur. Using the same nomenclature we could label this procedure as AB2F, "all but two fixed". This latter setup resembles traditional two state search models since they completely abstract from inactivity transitions. Under both decompositions we consider two scenarios: in the first one, we fix transition probabilities at their life-cycle means; in the second one, we set them to zero. ${ }^{19}$

Figure 12: Alternative Decomposition of life-cycle Unemployment rates, males
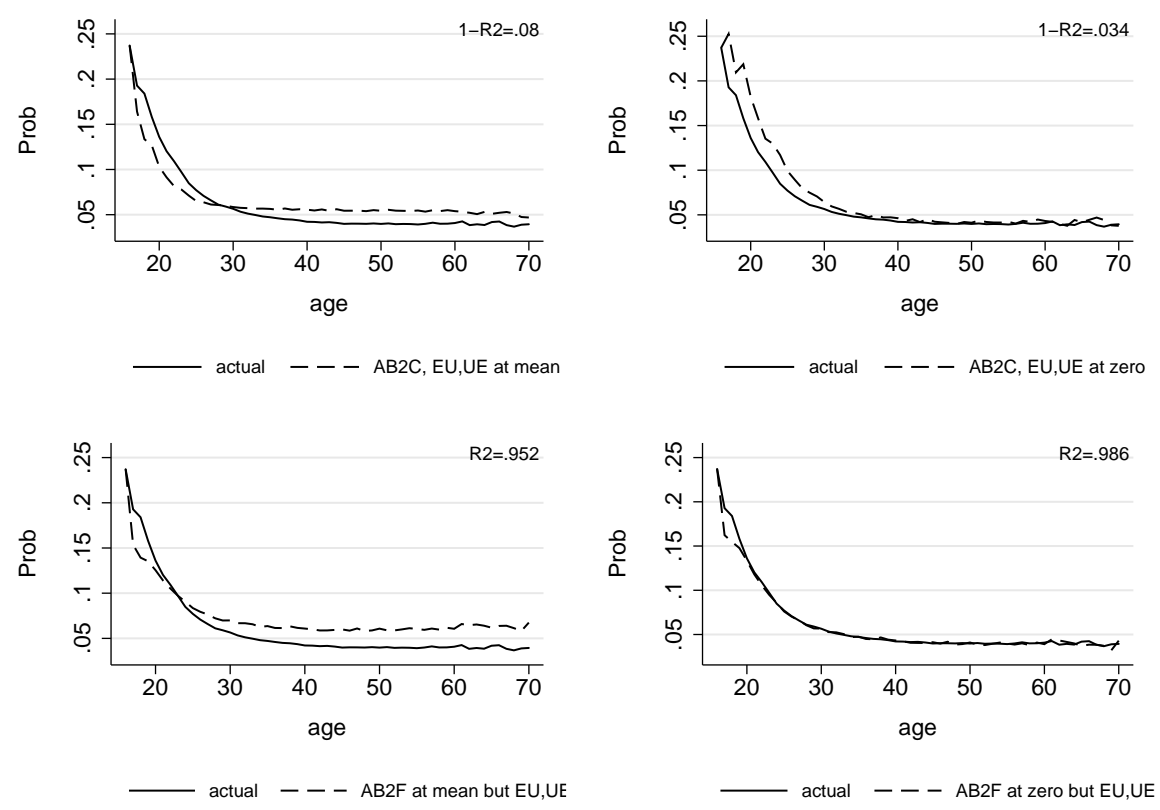

Note: Unconditional life-cycle profiles estimated via Seemingly Unrelated Regressions (SUR).

\footnotetext{
${ }^{19}$ However, it is more difficult to give a meaningful counterfactual interpretation to these exercises. We can think of our estimated transition probabilities as being the equilibrium outcomes from an underlying structural model. Thus, the more probabilities we change in our empirical counterfactual analysis, the more "equilibrium conditions" are implicitly changed, leading to adjustments that we cannot account for.
} 
Figure 13: Alternative Decomposition of life-cycle Unemployment rates, females
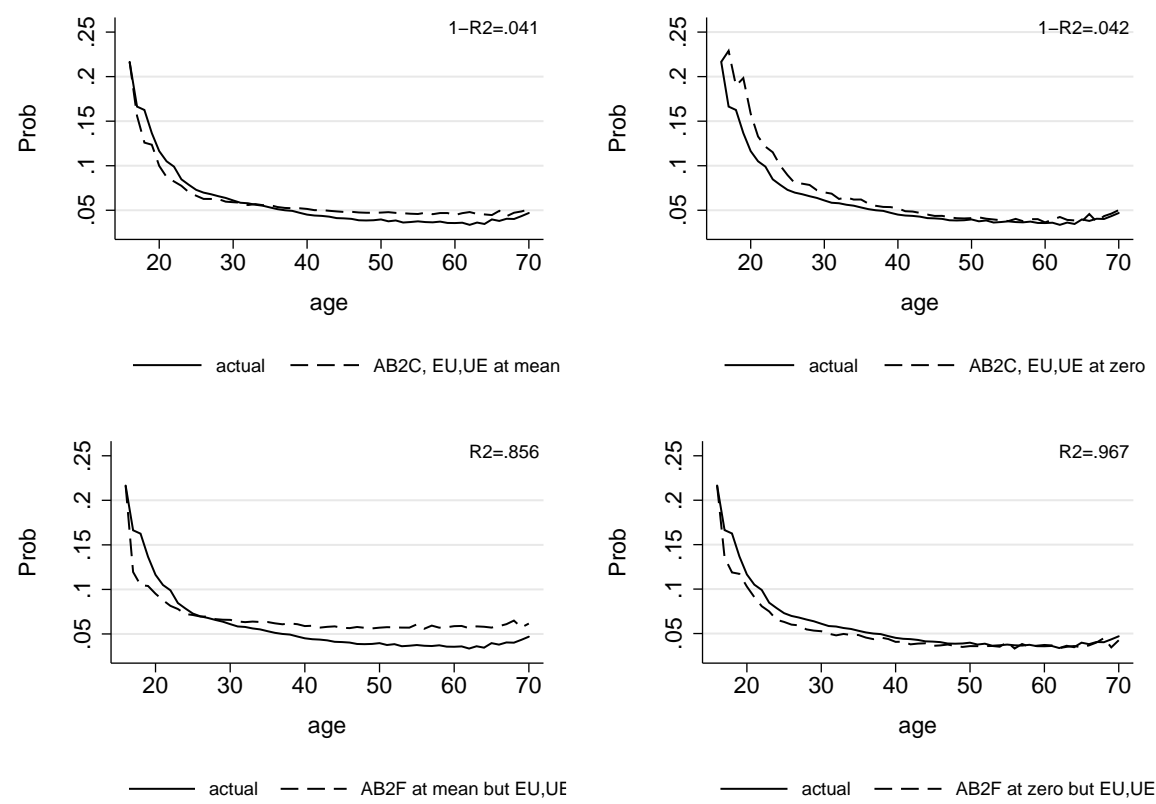

Note: Unconditional life-cycle profiles estimated via Seemingly Unrelated Regressions (SUR).

This rationale highlights a great danger in pursuing modeling strategies that ignore inactivity. As we see in Figures 12 and 13, the fit of the model in which inactivity transition probabilities are zero is nearly perfect for males and females (southeast subfigures). We could naively think that it is appropriate to omit inactivity from structural models as a modeling strategy. Even though such a model would likely give a good fit to the data, this approach has important shortcomings. First, the supposed accuracy is only true for averages (see the next section) leaving a great deal of withinage variation unaccounted. Second, such a model would be useless for running any counterfactual experiments and misleading for policy purposes. This is especially relevant if welfare implications are considered, since we would be omitting the welfare of agents transiting in or out of the labor force. If we perform a counterfactual experiment in a model with only $U E$ and $E U$ transitions, we are really doing two things: changing the aspect of interest of the model, and adjusting some unknown equilibrium conditions in order to keep inactivity transitions fixed at zero. Hence, the model structure is not invariant to policy changes. 


\subsection{Heterogeneity within age groups}

We now explore what would be the consequences of ignoring probability transitions in and out the labor force for the cross-sectional heterogeneity of unemployment and participation within age groups. We employ three methods that are in line with the methods we use for the differences between age groups.

The AB2C method keeps fixed only one flow at the estimated life-cycle profile. Here we focus on the $E U$ and $U E$ transitions, which are the only ones considered in standard two-state labor search models. Once we obtain a joint stochastic simulation of probability transitions for all flows, we substitute the particular profiles of $E U$ and $U E$ by their SUR estimated counterparts, the life-cycle "average" profile. Using the Markov chains and initial conditions at age 16, we learn how much variation in $U$ and $P$ is shut down at each age by keeping this two flows fixed. We report the ratio $Q_{a}^{A B 2 C,(E U, U E)}=1-\frac{\operatorname{Var}_{a}\left(U \mid \gamma^{E U}, \gamma^{U E}\right)}{\operatorname{Var}_{a}(U)}$.

The second method is called AB2F-mean. Accordingly, we stochastically simulate a joint lifecycle profile for all flows and then substitute all flows but the $E U$ and $U E$ transitions by their estimated SUR profiles. We run the Markov chain with these probabilities and obtain a "perturbed" life-cycle $U$ profile. We compute the variance of $U$ induced by these perturbed profiles and report the statistics $Q_{a}^{A B 2 F,-(E U, U E)}=\frac{\operatorname{Var}_{a}\left(U \mid \gamma^{E U}, \gamma^{U E}\right)}{\operatorname{Var}_{a}(U)}$.

We finally compute the method AB2F-0, which literally reflects what two-state labor market search models do: they constraint flows in and out of the labor force to be zero. We assess the contribution of $E U, U E$ flows as we did in the previous stage. For this method, we also report the implied variance when participation is ignored over $\operatorname{Var}_{a}(U)$.

Figure 20 reports the statistics calculated when applying each one of the three methods discussed above. Results are similar across exercises and basically indicate that when ignoring the participation decision, one is most likely to underestimate variances of unemployment over the life-cycle. The graphs suggest that the $E U$ and $U E$ transition probabilities are relevant for the variance of unemployment of young and prime aged, with a contribution that oscillates around $50 \%$ for women and $60 \%$ for men. However, the contribution is significantly lower for older workers: all statistics drop to a level below $20 \%$ at the end of the life-cycle. Nevertheless, the analysis shows that at every point of the lifecycle, a large proportion of the variance of unemployment (between 40 to $80 \%$ for males, and between 50 to $80 \%$ for females) is explained from variation of transitions 
Figure 14: Ignoring inactivity: unemployment variance decomposition by age group
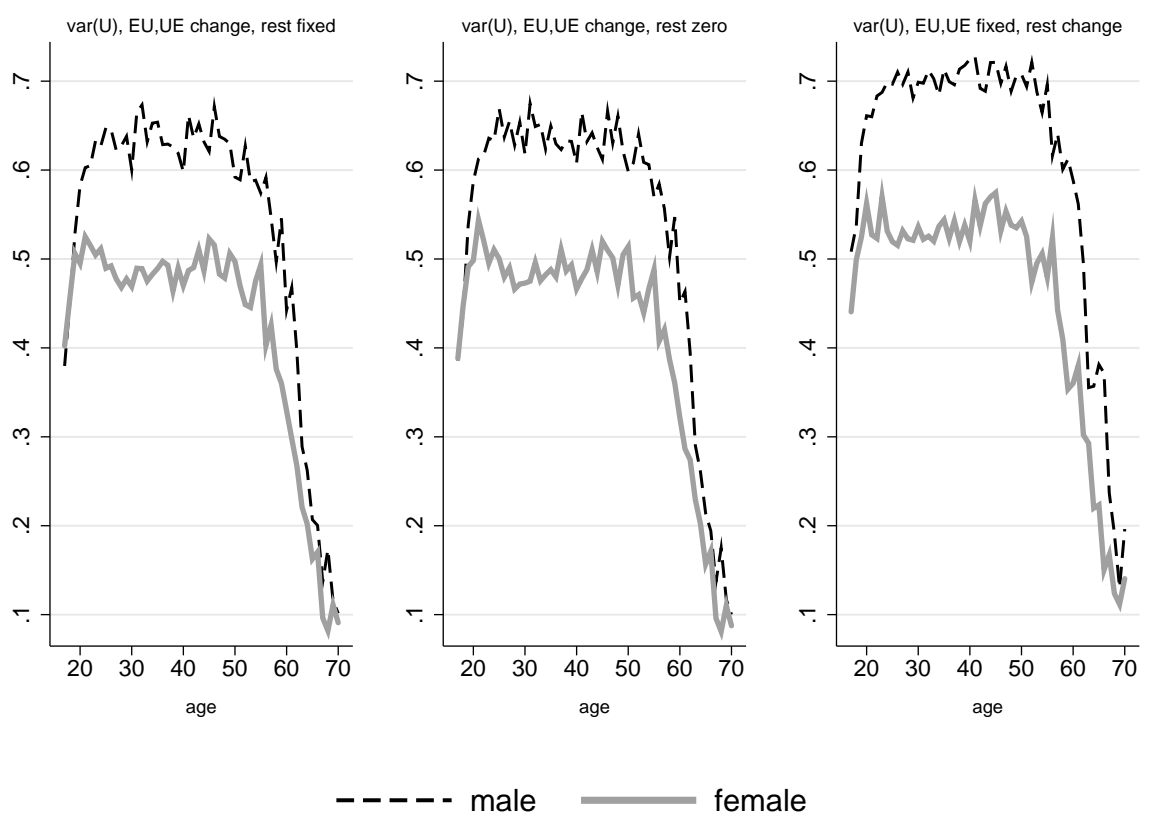

Note: Each figure reports $\operatorname{Var}\left(U_{a}\right)$ changes, based on 10000 simulations, SUR weighted unconditional estimates.

from and to inactivity. In addition, the joint contribution of $E U$ and $U E$ flows to explain unemployment variation does not depend on the particular profiles chosen (average lifecycle profiles or zero). This suggests that flows involving inactivity are a robust source of cross sectional variation for unemployment. Thus, when it comes to counterfactual experiments, any policy intervention with heterogeneous responses of inactivity-related transitions across the relevant population may induce significant effects in the cross-sectional unemployment responses. As an example, policy changes in the replacement ratio of an unemployment insurance program may increase the variability of individual unemployment not only through the usual channels as the job finding rate $U E$ or the separation rates EU. Our analysis suggests that you should not be surprised if $40 \%$ or $50 \%$ of the responses in unemployment comes from induced changes over decisions involving leaving or entering the labor force. Of course, a two-state labor market model would be forced to attribute most of this additional variability to either $E U$ or $U E$ flows. 


\section{Further analysis and relation to existing methods}

\subsection{Average Profiles}

Our decomposition method is similar to the one used by Pissarides (1986) and Shimer (2007). More specifically, unemployment and labor force participation approximations in the latter are the result of iterating the Markov chains an infinite number of times. Labor states obtained from twelve months of transitions (to simulate one year in the life of a worker) with empirical transition probabilities are not very different from the Markov chain limit. In most cases, the approximation is accurate so that we can construct theoretical counterparts to the observed proportion of individuals in each of the three considered states $\{e, u, o\}$ at age $a$ using the Markov chain limit. Therefore, the approximation at any age $a$ can be constructed by solving the following linear system ${ }^{20}$

$$
\begin{aligned}
\left(\gamma_{a}^{E U}+\gamma_{a}^{E O}\right) \widetilde{e}_{a} & =\gamma_{a}^{U E} \widetilde{u}_{a}+\gamma_{a}^{O E \widetilde{o}_{a}} \\
\left(\gamma_{a}^{U E}+\gamma_{a}^{U O}\right) \widetilde{u}_{a} & =\gamma_{a}^{E U} \widetilde{e}_{a}+\gamma_{a}^{O U} \widetilde{o}_{a} \\
\left(\gamma_{a}^{o e}+\gamma_{a}^{O U}\right) \widetilde{o}_{a} & =\gamma_{a}^{e O} \widetilde{e}_{a}+\gamma_{a}^{u o} \widetilde{u}_{a}
\end{aligned}
$$

The interpretation of these equations is straightforward. The left hand sides of these equations represent the flow of individuals transiting away from states $\{e, u, o\}$ respectively, at the end of age a. The right hand side accounts for the number of individuals transiting into those same states. These two numbers must be the same, assuming a stationary age-specific population structure and stationary transition probabilities $\gamma_{a}^{x z}$. Solving for the states, we get functional forms that relate them to age specific transition rates only.

$$
\begin{aligned}
\widetilde{e}_{a} & =\widetilde{e}\left(\gamma_{a}^{U E}, \gamma_{a}^{U O}, \gamma_{a}^{O E}, \gamma_{a}^{O U}\right) \\
\widetilde{u}_{a} & =\widetilde{u}\left(\gamma_{a}^{E U}, \gamma_{a}^{E O}, \gamma_{a}^{O E}, \gamma_{a}^{O U}\right) \\
\widetilde{o}_{a} & =\widetilde{o}\left(\gamma_{a}^{E U}, \gamma_{a}^{E O}, \gamma_{a}^{U E}, \gamma_{a}^{U O}\right)
\end{aligned}
$$

accordingly, we can construct these "theoretical" counterparts for participation $\left(\widetilde{P}_{a}=1-\widetilde{o}_{a}\right)$ and unemployment rates $\left(\widetilde{U}_{a}=\widetilde{u}_{a} /\left(\widetilde{e}_{a}+\widetilde{u}_{a}\right)\right)$ using the above equations and our estimates of $\left\{\gamma_{a}^{x z}\right\}$

\footnotetext{
${ }^{20}$ The limiting labor states $e, u, o$ are just the normalized eigenvector (so that its components add up to 1 ) associated to an eigenvalue of value 1 .
} 
from regression (1). In Figure 15 we plot the observed versus theoretical (constructed) rates, for both men and women.

Figure 15: Participation and Unemployment rates: actual vs. limit of Markov
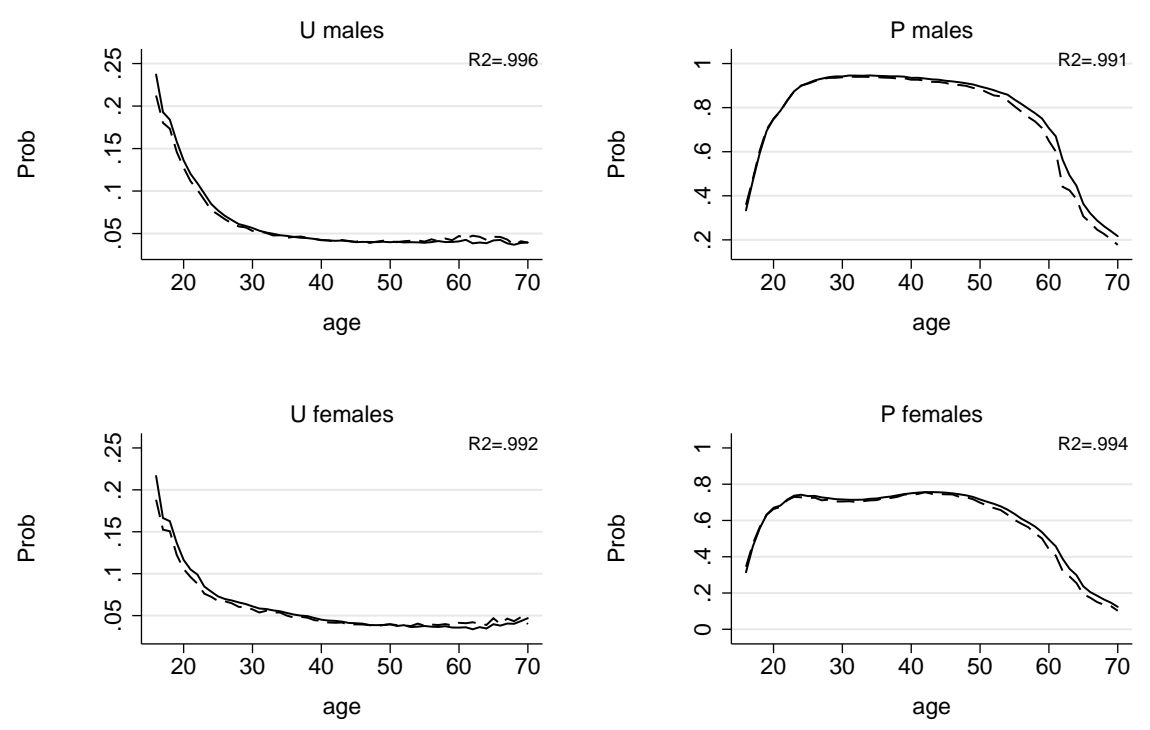

actual $\quad$ - - - - - lim Markov

Note: Unconditional life-cycle profiles estimated via Seemingly Unrelated Regressions (SUR).

As seen from the Figure, the theoretical rates follow closely their observed counterparts and pose a reasonable approximation to the observed profiles. Notice that in order to calculate stocks of unemployed, employed and inactive workers, the method above does not rely on initial conditions/distribution of workers across employment states but only age-specific transition probabilities. The goodness of fit of the theoretical rates is due to high monthly transition probabilities, which dwarfs the effect of initial conditions.

Given that theoretical participation and unemployment rates depend only on age-specific transition probabilities, we can assess their relative importance in explaining aggregate life-cycle profiles. Using the same logic as in the "all but one change" (AB1C) method, ${ }^{21}$ we compute the limiting states at each age by using our estimates $\gamma_{a}^{x z}$. However, we keep fixed a particular transition probability at its mean life-cycle value, one at a time, and we allow the rest of them to change according

\footnotetext{
${ }^{21}$ This is in contrast to what Shimer (2007) does in the context of a business cycle decomposition. He fixes all transition probabilities at their mean and changes only one, what we labeled the AB1F method above.
} 
to age. We present these decompositions for unemployment and participation in Figures 17 and 19 below.

Figure 16: Decomposition of life-cycle Unemployment, limit Markov for males
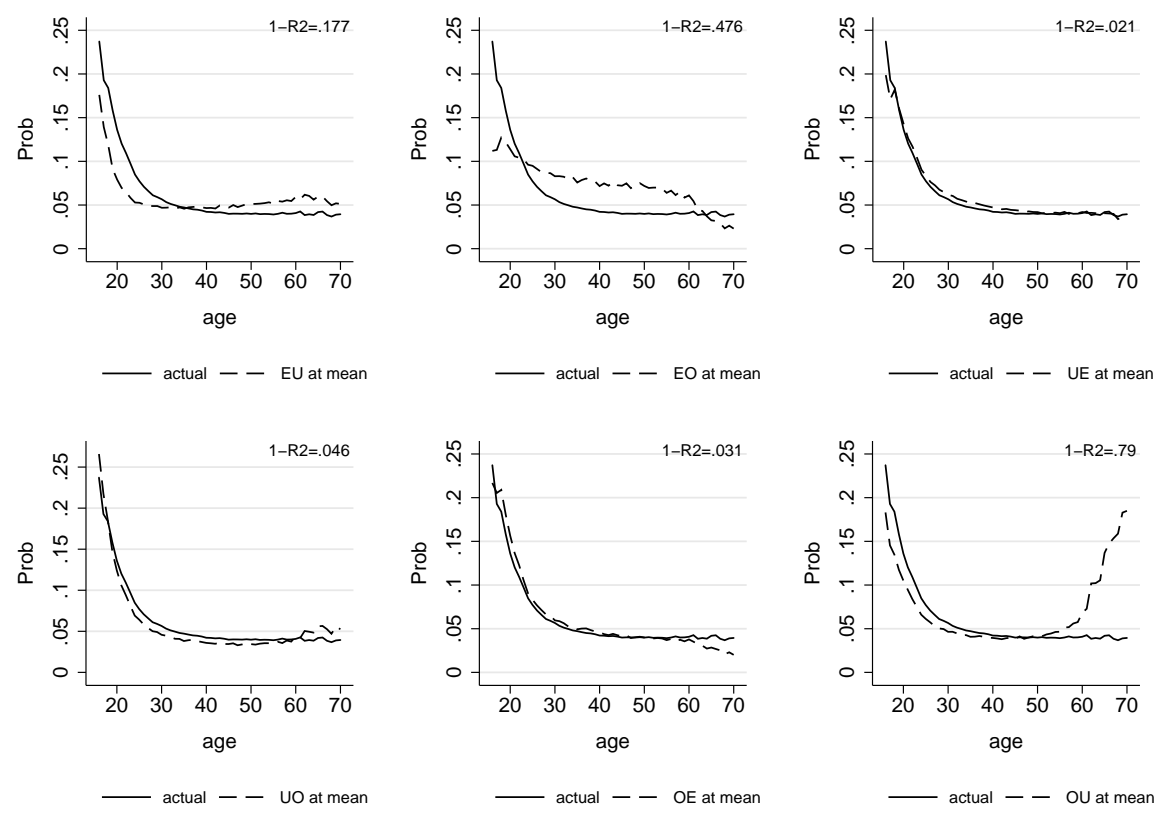

Note: Unconditional life-cycle profiles estimated via Seemingly Unrelated Regressions (SUR).

When comparing the results from this "limit" method to the ones we see in the Markov chain analysis, we get roughly identical results. In terms of participation, the most important transition probability is the one from employment to inactivity $E O$. If this transition probability were to be constant throughout the life-cycle, the participation profile would be flatter. The $E O$ probability is very important to determine early and late life employment status. Also, movements from inactivity into the labor force (both $O E$ and $O U$ probabilities) determine to a great extent unemployment after the age of 60 .

As for the life-cycle profile of the unemployment rate, again the $E O$ probability plays an important role, followed by the $E U$ as well as the $O U$ transition probabilities. The job finding probability $(U E)$ does not affect differences in life-cycle participation and unemployment significantly. It turns out that the transitions into and out from the labor force are quite important in shaping unemployment and participation rates life-cycle profiles. 
Figure 17: Decomposition of life-cycle Unemployment, limit Markov for females
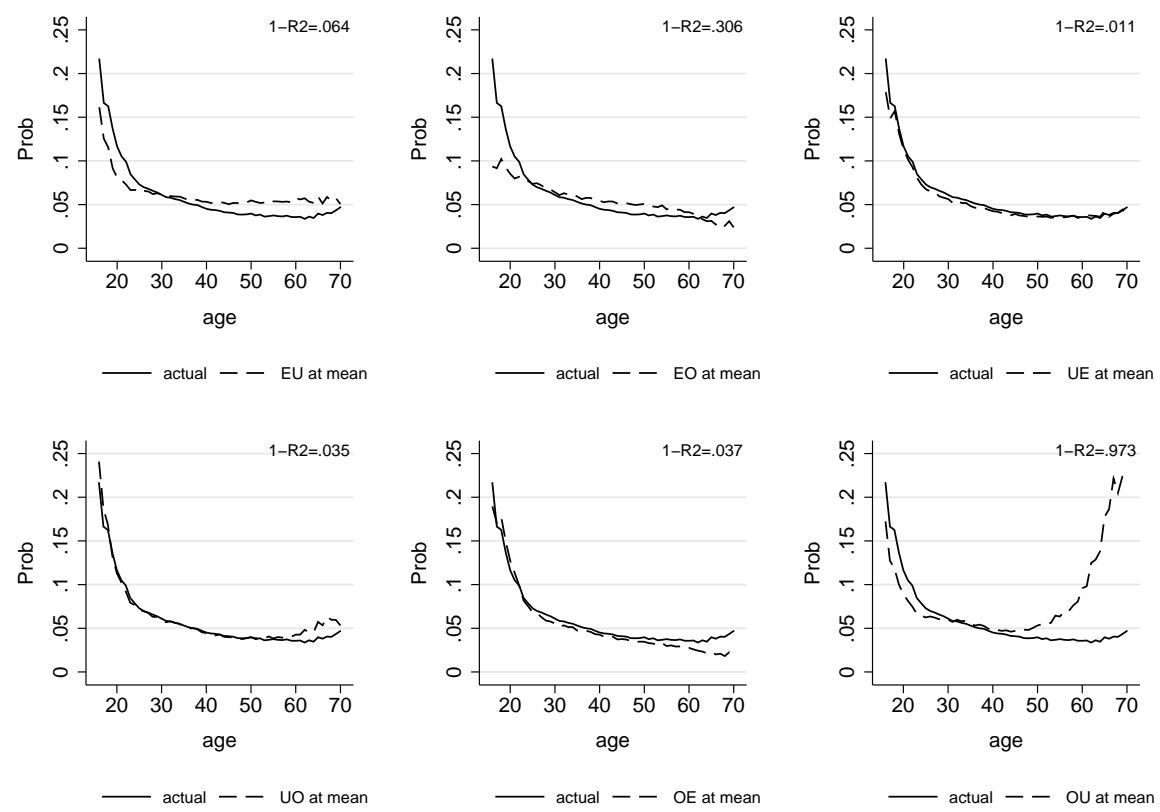

Note: Unconditional life-cycle profiles estimated via Seemingly Unrelated Regressions (SUR).

These results contrasts to Shimer (2007) and Fujita and Ramey (2009) findings in relation to the business cycle. These authors show that $U E$ and $E U$ flows are enough to account for the cyclical fluctuations of the unemployment rate. For life-cycle analysis, our evidence shows that inactivity transitions are key to understand the unemployment and participation by age.

\subsection{Heterogeneity within age groups}

The Pissarides-Shimer decomposition can also be applied to extend the analysis of Section 4.2. Specifically it allows to separate the statistics depicted in Figures 10 and 11 as the product of two components. The first component is the variance of each $\left\{\gamma_{a}^{x z}\right\}$, while the second component is the marginal effect of the variance in unemployment or participation with respect to each transition probability, which we denote by $\frac{\partial \widetilde{U}_{a}}{\partial \gamma_{a}^{z z}}$ in the case of unemployment and $\frac{\partial \widetilde{P}_{a}}{\partial \gamma_{a}^{x z}}$ in the case of participation. By using the limit of the Markov chain and a first-order Taylor expansion, we obtain an approximation of the marginal effect for each age group. Those are reported on Figures 20 and 21. ${ }^{22}$ Comparing these Figures with Figures 10 and 11 suggests that for the case of unemployment

\footnotetext{
${ }^{22}$ More details on how these semi elasticities are approximated are given in the Appendix.
} 
Figure 18: Decomposition of life-cycle Participation, limit Markov for males
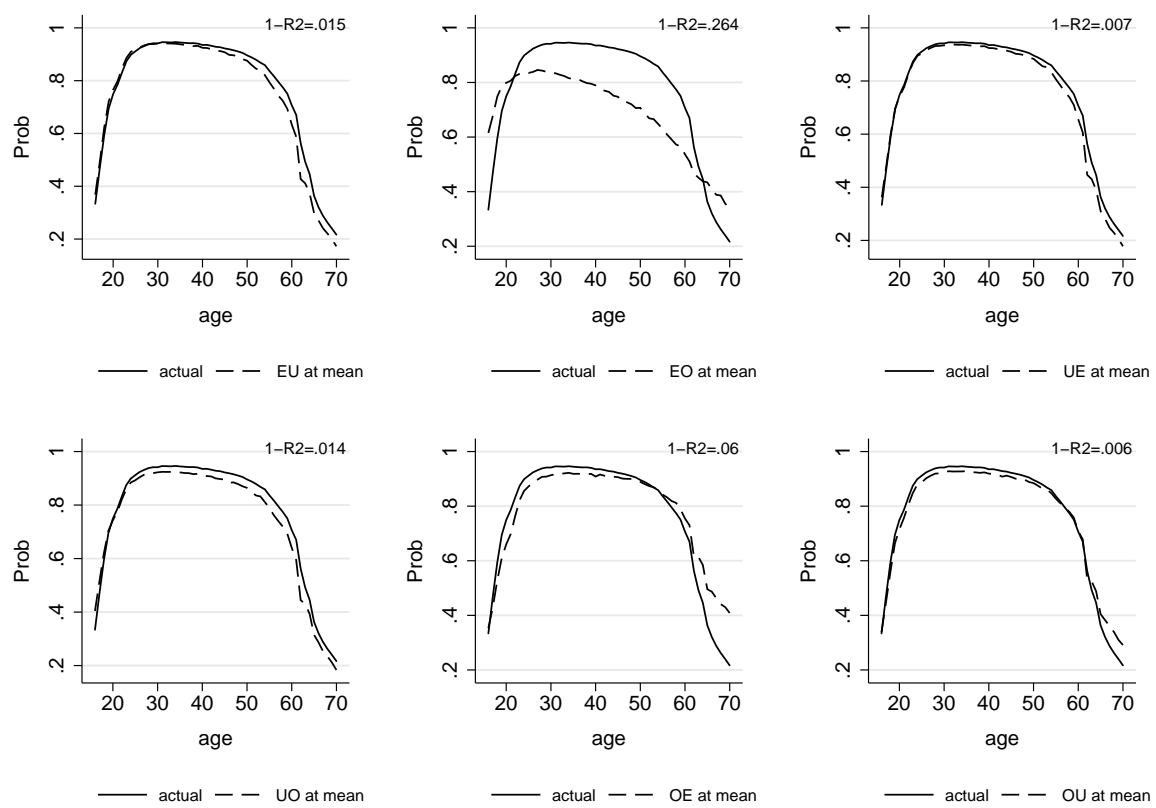

Note: Unconditional life-cycle profiles estimated via Seemingly Unrelated Regressions (SUR).

profiles, the variability of each flow is the main indicator of its influence, given that the computed semi elasticities are constant and small over the life-cycle (with the exception of $O U$ probabilities). On the other hand, participation is influenced in a mixed way by the transition probabilities: $E U$, $E O, O E$ and $O U$ exhibit non-constant profiles of semi elasticities over the life-cycle, while those related to $U E$ and $U O$ are flat and almost equal to zero.

\section{Conclusion}

In this paper we estimate and report life cycle transition probabilities across labor market states for male and female workers in the US, using data from the Current Population Population Survey. We construct measures of worker flows between labor force states according to age. This procedure gives us a consistent set of facts from which we can identify age dependent job finding and job destruction rates as well as labor force exit/entry rates.

Using our estimates, we find that most differences in participation and unemployment rates over the life-cycle, both in terms of averages and variances, can be attributed to the probability of 
Figure 19: Decomposition of life-cycle Participation, limit Markov for females
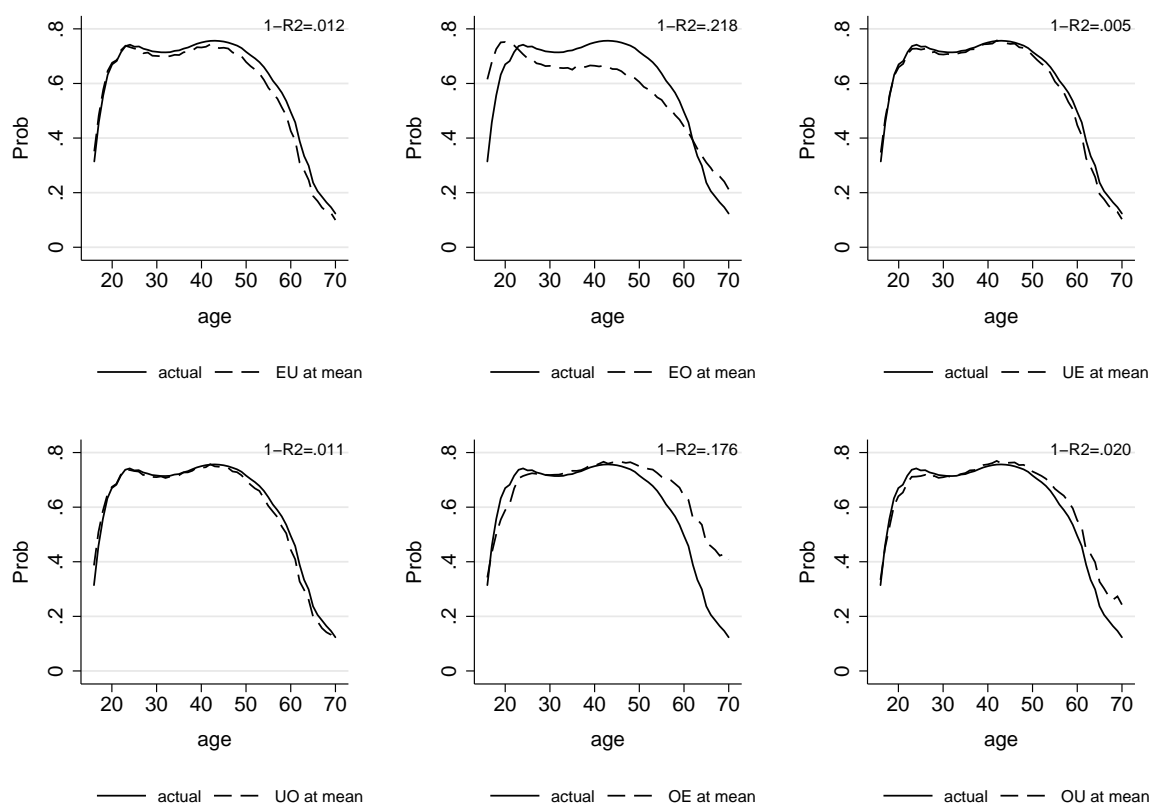

Note: Unconditional life-cycle profiles estimated via Seemingly Unrelated Regressions (SUR).

leaving employment and the probability of entering unemployment from inactivity. On the other hand, the job finding rate plays a role explaining heterogeneity but explains little of the life-cycle unemployment average profile.

We argue that two-state labor market models are not appropriate to perform counterfactual experiments for policy analysis even though they may provide a good fit to unemployment facts. The data shows a great deal of inactivity transitions, whose variation greatly affects unemployment and participation life-cycle profiles. In our analysis of the unemployment and participation variation by age, we find that inactivity related flows $(E O, U O, O E$, and $O U)$ jointly account for a sizable amount of dispersion, ranging from $40 \%$ (for prime-aged males) to $80 \%$ (old males and females). If a model ignores flows in and out of inactivity, any policy experiment in such artificial economy would also involve an unknown change of general equilibrium conditions in order to maintain inactivity transition probabilities fixed at zero. Even though one may incorporate inactivity as a labor force entry during youth and a retirement decision, the large amount of transitions into and out from inactivity for prime-aged workers suggests that such an approach may be insufficient. In 
Figure 20: Within-group variability: unemployment marginal effects
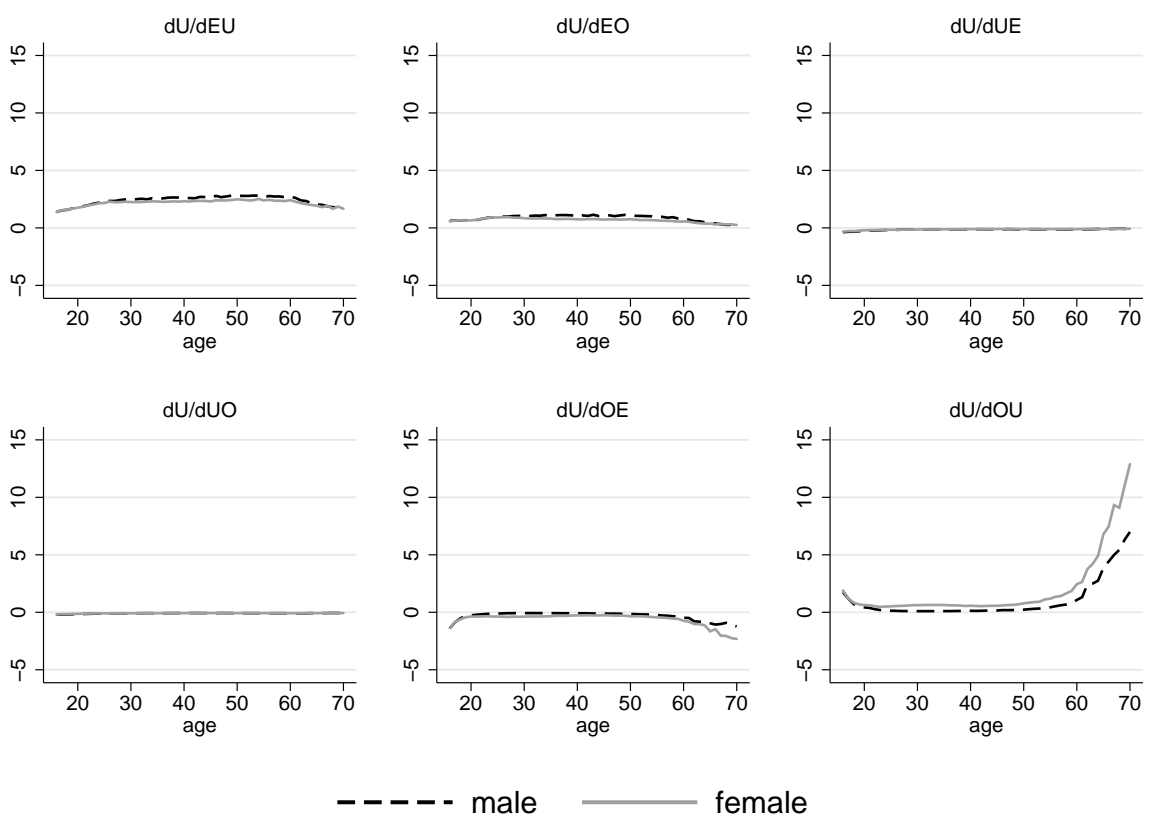

Note: Figures show approximated derivative $\partial U / \partial \gamma$, where $\gamma$ are the unconditional life-cycle profiles estimated via Seemingly Unrelated Regressions (SUR).

the case of female workers, fertility and child rearing considerations makes our recommendation even more valid. Indeed, it is quite suggestive that the transitions explaining most of unemployment and participation levels and variations are the same for both males and females, as shown in our analysis.

Our main conclusion is that further study of job market experiences over the life-cycle should always include the inactivity state and endogenous participation decisions. Not doing so would bias the imputed importance of other factors determining participation and unemployment during the life-cycle and thus, aggregate participation and unemployment rates. 
Figure 21: Within-group variability: participation marginal effects
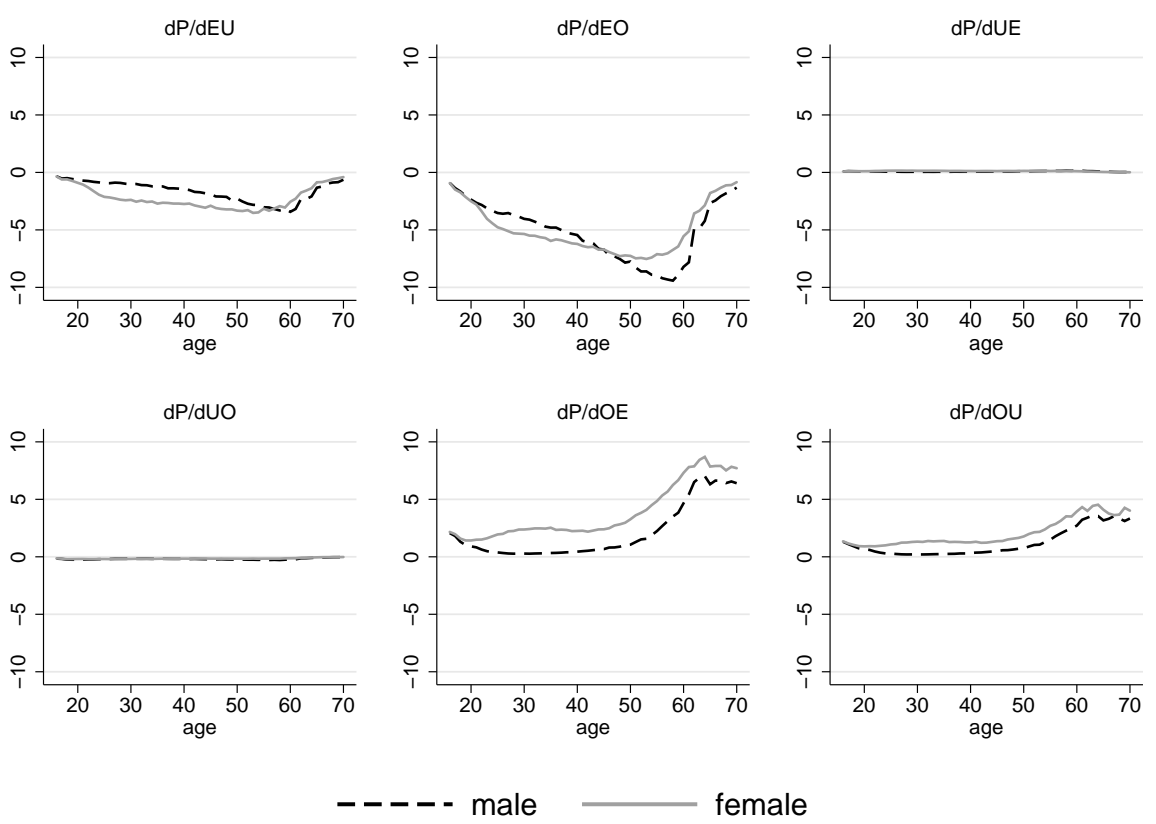

Note: Figures show approximated derivative $\partial P / \partial \gamma$, where $\gamma$ are the unconditional life-cycle profiles estimated via Seemingly Unrelated Regressions (SUR).

\section{References}

Abowd, J. M., And A. Zellner (1985): "Estimating Gross Labor-Force Flows," Journal of Business \& Economic Statistics, 3(3), 254-83.

Alvarez, F., And M. Veracierto (1999): "Labor-market Policies in an Equilibrium Search Model," NBER Macroeconomics Annuals, pp. pp. 265-304.

Blanchard, O. J., and P. Diamond (1990): "The Cyclical Behavior of the Gross Flows of U.S. Workers," Brookings Papers on Economic Activity, 21(1990-2), 85-156.

Chéron, A., J. Hairault, and F. Langot (2008): "Life-Cycle Equilibrium Unemployment," Discussion paper no. 3396, IZA.

(2011): “Age-dependent employment protection," Economic Journal, 121(557), pp. 14771504.

Darby, M. R., J. C. Haltiwanger, and M. W. Plant (1986): "The Ins and Outs of Unemployment: The Ins Win," UCLA Economics Working Papers 411, UCLA Department of Economics.

Davis, S. J. (1987): "Fluctuations in the Pace of Labor Reallocation," Carnegie-Rochester Conference Series on Public Policy, 27(1), 335-402.

Elsby, M. W. L., R. Michaels, and G. Solon (2009): "The Ins and Outs of Cyclical Unemployment," American Economic Journal: Macroeconomics, 1(1), 84-110. 
Fujita, S., And G. Ramey (2009): "The Cyclicality of Separation and Job Finding Rates," International Economic Review, 5(2), pp. 415-430.

Garibaldi, P., And E. Wasmer (2005): "Equilibrium Search Unemployment, Endogenous Participation, and Labor Market Flows," Journal of the European Economic Association, 3(4), 851-882.

Hairault, J.-O., F. Langot, and T. Sopraseuth (2010): "Distance to Retirement and Older Workers' Employment: The Case For Delaying The Retirement Age," Journal of the European Economic Association, 8(5), 1034-1076.

Hall, R. E. (2006): "Job Loss, Job Finding and Unemployment in the U.S. Economy over the Past 50 Years," in NBER Macroeconomics Annual 2005, Volume 20, NBER Chapters, pp. 101-166. National Bureau of Economic Research, Inc.

Jaimovich, N., And H. Siu (2009): "The Young, the Old, and the Restless: Demographics and Business Cycle Volatility," American Economic Review, 99(3), pp. 804-826.

Kitao, S., L. Luunqvist, and T. Sargent (2008): "A Life Cycle Model of Trans-Atlantic Employment Experiences," mimeo, University of Southern California.

Krussel, P., T. Mukoyama, R. Rogerson, and A. Şahin (2008): "Aggregate Implications of Indivisible labor, incomplete markets, and labor market frictions," Journal of Monetary Economics, 55, 961-979.

- (2010): "Aggregate labor market outcomes: the roles of choice and chance," Quantitative Economics, 1(1), 97-127.

LuUngqvist, L., And T. SArgent (2008): "Two questions about European unemployment," Econometrica, 76(1), pp. 1-29.

Menzio, G., I. Telyukova, And L. Visschers (2010): "Directed Search over the Life-Cycle," mimeo, University of Pennsylvania.

Michelacci, C., and H. Ruffo (2010): "Optimal life cycle unemployment," Discussion paper, CEMFI.

OECD (1996): "Growing into Work," in Employment Outlook, chap. 4, pp. 109-159. OECD.

(1998): "Work-Force Ageing in OECD Countries," in Employment Outlook, chap. 4, pp. 123-151. OECD.

Pissarides, C. A. (1986): "Unemployment and Vacancies in Britain," Economic Policy, 1(3), 499-559.

Pries, M., and R. Rogerson (2009): "Search Frictions and Labor Market Participation," European Economic Review, 53, pp. 568-587.

Ravn, M. O. (2006): "The Consumption - Tightness Puzzle," NBER International Seminar on Macroeconomics, pp. pp. 9-63. 
Rios-Rull, J.-V. (1996): "Life-Cycle Economies and Aggregate Fluctuations," The Review of Economic Studies, 63(3), pp. 465-489.

Rogerson, R. (2008): "Structural Transformation and the Deterioration of European Labor Market Outcomes," Journal of Political Economy, 116(2), pp. 235-259.

SHIMER, R. (2007): "Reassessing the Ins and Outs of Unemployment," NBER Working Papers 13421, National Bureau of Economic Research, Inc.

Veracierto, M. (2008): "On the Cyclical Behavior of Employment, Unemployment and Labor Force Participation," Journal of Monetary Economics, 55(6), 1143-1157. 


\section{A Appendix}

\section{A.1 Approximation of variance and its decomposition}

Using the limit of the order-one Markov chain, we can obtain an approximation for the unemployment and participation rates conditional on age as in Shimer (2007). In this way, we obtain

$$
\begin{aligned}
U_{a} & \approx \widetilde{U}_{a}=\frac{\gamma_{a}^{o e} \gamma_{a}^{e u}+\gamma_{a}^{o u}\left(\gamma_{a}^{e u}+\gamma_{a}^{e o}\right)}{\gamma_{a}^{o e}\left(\gamma_{a}^{u o}+\gamma_{a}^{e u}\right)+\gamma_{a}^{u e}\left(\gamma_{a}^{o e}+\gamma_{a}^{o u}\right)+\gamma_{a}^{o u}\left(\gamma_{a}^{e u}+\gamma_{a}^{e o}\right)} \\
P_{a} \approx \widetilde{P}_{a} & =\frac{\gamma_{a}^{u e}\left(\gamma_{a}^{o e}+\gamma_{a}^{o u}\right)+\gamma_{a}^{o e}\left(\gamma_{a}^{u o}+\gamma_{a}^{e u}\right)+\gamma_{a}^{o u}\left(\gamma_{a}^{e u}+\gamma_{a}^{e o}\right)}{\gamma_{a}^{u e} \gamma_{a}^{e o}+\gamma_{a}^{e o} \gamma_{a}^{u o}+\gamma_{a}^{u o} \gamma_{a}^{e u}+\gamma_{a}^{u e}\left(\gamma_{a}^{o e}+\gamma_{a}^{o u}\right)+\gamma_{a}^{o e}\left(\gamma_{a}^{u o}+\gamma_{a}^{e u}\right)+\gamma_{a}^{o u}\left(\gamma_{a}^{e u}+\gamma_{a}^{e o}\right)}
\end{aligned}
$$

From these approximations, it follows a variance decomposition that is similar to Fujita and Ramey (2009) and Elsby, Michaels, and Solon (2009) although they do it in terms of unemployment variation instead of unemployment and participation levels. First, we approximate these unemployment and participation via a first-order Taylor expansion around the age conditional mean of each flow, $\bar{\gamma}_{a} \equiv\left\{\bar{\gamma}_{a}^{x z}\right\}$ for $x, z \in\{e, u, o\}$.

$$
\widetilde{U}_{a} \approx \widetilde{U}_{a}\left(\bar{\gamma}_{a}\right)+\sum_{x} \sum_{z \neq x} \frac{\partial \widetilde{U}_{a}}{\partial \gamma_{a}^{x z}}\left(\bar{\gamma}_{a}\right)\left(\gamma_{a}^{x z}-\bar{\gamma}_{a}^{x z}\right)
$$

Once we linearize, we compute the corresponding variance of $\widetilde{U}_{a}$ and

$$
\mathbb{V}\left[\widetilde{U}_{a}\right] \approx \sum_{x} \sum_{z \neq x}\left(\frac{\partial \widetilde{U}_{a}}{\partial \gamma_{a}^{x z}}\left(\bar{\gamma}_{a}\right)\right)^{2} \mathbb{V}\left[\gamma_{a}^{x z}\right]+2 \sum_{x} \sum_{z \neq x} \sum_{y \neq x} \sum_{w \neq y} \frac{\partial \widetilde{U}_{a}}{\partial \gamma_{a}^{x z}}\left(\bar{\gamma}_{a}\right) \frac{\partial \widetilde{U}_{a}}{\partial \gamma_{a}^{y w}}\left(\bar{\gamma}_{a}\right) \mathbb{C O V}\left[\gamma_{a}^{x z}, \gamma_{a}^{y w}\right]
$$

As seen from the expression above, the approximated rate of unemployment $\widetilde{U}_{a}$ and its variance $\mathbb{V}\left[\widetilde{U}_{a}\right]$ are affected directly by all marginal effects $\frac{\partial \widetilde{U}_{a}}{\partial \gamma_{a}^{x z}}$. The same procedure trivially applies to the approximated participation $\widetilde{P}_{a}$.

The specific partial derivatives are

$$
\begin{aligned}
\frac{\partial \widetilde{U}_{a}}{\partial \gamma_{a}^{e u}} & =\widetilde{U}_{a}\left(1-\widetilde{U}_{a}\right) \frac{\gamma_{a}^{o e}+\gamma_{a}^{o u}}{\gamma_{a}^{e u} \gamma_{a}^{o e}+\gamma_{a}^{e o} \gamma_{a}^{o u}+\gamma_{a}^{e u} \gamma_{a}^{o u}} \\
\frac{\partial \widetilde{U}_{a}}{\partial \gamma_{a}^{e o}} & =\widetilde{U}_{a}\left(1-\widetilde{U}_{a}\right) \frac{\gamma_{a}^{o u}}{\gamma_{a}^{e u} \gamma_{a}^{o e}+\gamma_{a}^{e o} \gamma_{a}^{o u}+\gamma_{a}^{e u} \gamma_{a}^{o u}} \\
\frac{\partial \widetilde{U}_{a}}{\partial \gamma_{a}^{u e}} & =-\widetilde{U}_{a}\left(1-\widetilde{U}_{a}\right) \frac{\gamma_{a}^{o e}+\gamma_{a}^{o u}}{\gamma_{a}^{o e} \gamma_{a}^{u e}+\gamma_{a}^{o e} \gamma_{a}^{u o}+\gamma_{a}^{o u} \gamma_{a}^{u e}} \\
\frac{\partial \widetilde{U}_{a}}{\partial \gamma_{a}^{u o}} & =-\widetilde{U}_{a}\left(1-\widetilde{U}_{a}\right) \frac{\gamma_{a}^{o e}}{\gamma_{a}^{o e} \gamma_{a}^{u e}+\gamma_{a}^{o e} \gamma_{a}^{u o}+\gamma_{a}^{o u} \gamma_{a}^{u e}} \\
\frac{\partial \widetilde{U}_{a}}{\partial \gamma_{a}^{o e}} & =-\widetilde{U}_{a}\left(1-\widetilde{U}_{a}\right) \frac{\gamma_{a}^{o u}\left(\gamma_{a}^{e o} \gamma_{a}^{u e}+\gamma_{a}^{e o} \gamma_{a}^{u o}+\gamma_{a}^{e u} \gamma_{a}^{u o}\right)}{\left(\gamma_{a}^{o e} \gamma_{a}^{u e}+\gamma_{a}^{o e} \gamma_{a}^{u o}+\gamma_{a}^{o u} \gamma_{a}^{u e}\right)\left(\gamma_{a}^{e u} \gamma_{a}^{o e}+\gamma_{a}^{e o} \gamma_{a}^{o u}+\gamma_{a}^{e u} \gamma_{a}^{o u}\right)} \\
\frac{\partial \widetilde{U}_{a}}{\partial \gamma_{a}^{o u}} & =\widetilde{U}_{a}\left(1-\widetilde{U}_{a}\right) \frac{\gamma_{a}^{o e}\left(\gamma_{a}^{e o} \gamma_{a}^{u e}+\gamma_{a}^{e o} \gamma_{a}^{u o}+\gamma_{a}^{e u} \gamma_{a}^{u o}\right)}{\left(\gamma_{a}^{o e} \gamma_{a}^{u e}+\gamma_{a}^{o e} \gamma_{a}^{u o}+\gamma_{a}^{o u} \gamma_{a}^{u e}\right)\left(\gamma_{a}^{e u} \gamma_{a}^{o e}+\gamma_{a}^{e o} \gamma_{a}^{o u}+\gamma_{a}^{e u} \gamma_{a}^{o u}\right)}
\end{aligned}
$$


For participation, we have that

$$
\begin{aligned}
\frac{\partial \widetilde{P}_{a}}{\partial \gamma_{a}^{e u}} & =\widetilde{P}_{a}\left(1-\widetilde{P}_{a}\right) \frac{\left(\gamma_{a}^{e o}-\gamma_{a}^{u o}\right)\left(\gamma_{a}^{o e} \gamma_{a}^{u e}+\gamma_{a}^{o e} \gamma_{a}^{u o}+\gamma_{a}^{o u} \gamma_{a}^{u e}\right)}{\left(\gamma_{a}^{e o} \gamma_{a}^{u e}+\gamma_{a}^{e o} \gamma_{a}^{u o}+\gamma_{a}^{e u} \gamma_{a}^{u o}\right)\left(\gamma_{a}^{e u} \gamma_{a}^{o e}+\gamma_{a}^{e o} \gamma_{a}^{o u}+\gamma_{a}^{e u} \gamma_{a}^{o u}+\gamma_{a}^{o e} \gamma_{a}^{u e}+\gamma_{a}^{o e} \gamma_{a}^{u o}+\gamma_{a}^{o u} \gamma_{a}^{u e}\right)} \\
\frac{\partial \widetilde{P}_{a}}{\partial \gamma_{a}^{e o}} & =\widetilde{P}_{a}\left(1-\widetilde{P}_{a}\right)-\frac{\left(\gamma_{a}^{o e} \gamma_{a}^{u e}+\gamma_{a}^{o e} \gamma_{a}^{u o}+\gamma_{a}^{o u} \gamma_{a}^{u e}\right)\left(\gamma_{a}^{e u}+\gamma_{a}^{u e}+\gamma_{a}^{u o}\right)}{\left(\gamma_{a}^{e o} \gamma_{a}^{u e}+\gamma_{a}^{e o} \gamma_{a}^{u o}+\gamma_{a}^{e u} \gamma_{a}^{u o}\right)\left(\gamma_{a}^{e u} \gamma_{a}^{o e}+\gamma_{a}^{e o} \gamma_{a}^{o u}+\gamma_{a}^{e u} \gamma_{a}^{o u}+\gamma_{a}^{o e} \gamma_{a}^{u e}+\gamma_{a}^{o e} \gamma_{a}^{u o}+\gamma_{a}^{o u} \gamma_{a}^{u e}\right)} \\
\frac{\partial \widetilde{P}_{a}}{\partial \gamma_{a}^{u e}} & =-\widetilde{P}_{a}\left(1-\widetilde{P}_{a}\right) \frac{\left(\gamma_{a}^{e o}-\gamma_{a}^{u o}\right)\left(\gamma_{a}^{e u} \gamma_{a}^{o e}+\gamma_{a}^{e o} \gamma_{a}^{o u}+\gamma_{a}^{e u} \gamma_{a}^{o u}\right)}{\left(\gamma_{a}^{e o} \gamma_{a}^{u e}+\gamma_{a}^{e o} \gamma_{a}^{u o}+\gamma_{a}^{e u} \gamma_{a}^{u o}\right)\left(\gamma_{a}^{e u} \gamma_{a}^{o e}+\gamma_{a}^{e o} \gamma_{a}^{o u}+\gamma_{a}^{e u} \gamma_{a}^{o u}+\gamma_{a}^{o e} \gamma_{a}^{u e}+\gamma_{a}^{o e} \gamma_{a}^{u o}+\gamma_{a}^{o u} \gamma_{a}^{u e}\right)} \\
\frac{\partial \widetilde{P}_{a}}{\partial \gamma_{a}^{u o}} & =-\widetilde{P}_{a}\left(1-\widetilde{P}_{a}\right) \frac{\left(\gamma_{a}^{e o}+\gamma_{a}^{e u}+\gamma_{a}^{u e}\right)\left(\gamma_{a}^{e u} \gamma_{a}^{o e}+\gamma_{a}^{e o} \gamma_{a}^{o u}+\gamma_{a}^{e u} \gamma_{a}^{o u}\right)}{\left(\gamma_{a}^{e o} \gamma_{a}^{u e}+\gamma_{a}^{e o} \gamma_{a}^{u o}+\gamma_{a}^{e u} \gamma_{a}^{u o}\right)\left(\gamma_{a}^{e u} \gamma_{a}^{o e}+\gamma_{a}^{e o} \gamma_{a}^{o u}+\gamma_{a}^{e u} \gamma_{a}^{o u}+\gamma_{a}^{o e} \gamma_{a}^{u e}+\gamma_{a}^{o e} \gamma_{a}^{u o}+\gamma_{a}^{o u} \gamma_{a}^{u e}\right)} \\
\frac{\partial \widetilde{P}_{a}}{\partial \gamma_{a}^{o e}} & =\widetilde{P}_{a}\left(1-\widetilde{P}_{a}\right) \frac{\gamma_{a}^{e u}+\gamma_{a}^{u e}+\gamma_{a}^{u o}}{\gamma_{a}^{e u} \gamma_{a}^{o e}+\gamma_{a}^{e o} \gamma_{a}^{o u}+\gamma_{a}^{e u} \gamma_{a}^{o u}+\gamma_{a}^{o e} \gamma_{a}^{u e}+\gamma_{a}^{o e} \gamma_{a}^{u o}+\gamma_{a}^{o u} \gamma_{a}^{u e}} \\
\frac{\partial \widetilde{P}_{a}}{\partial \gamma_{a}^{o e}} & =\widetilde{P}_{a}\left(1-\widetilde{P}_{a}\right) \frac{\gamma_{a}^{e o}+\gamma_{a}^{e u}+\gamma_{a}^{u e}}{\gamma_{a}^{e u} \gamma_{a}^{o e}+\gamma_{a}^{e o} \gamma_{a}^{o u}+\gamma_{a}^{e u} \gamma_{a}^{o u}+\gamma_{a}^{o e} \gamma_{a}^{u e}+\gamma_{a}^{o e} \gamma_{a}^{u o}+\gamma_{a}^{o u} \gamma_{a}^{u e}}
\end{aligned}
$$




\section{A.2 Robustness [NOT FOR PUBLICATION]}

In this appendix we present the same figures as in the body of the paper, but where our estimates include controls for time, cohort and state variation in matrix $D$ below:

$$
f_{a t c}^{x z} \sqrt{N_{a t c}^{x}}=\sum_{a=1}^{A} \gamma_{a}^{x z} D_{a t c} \sqrt{N_{a t c}^{x}}+\beta W_{a t c} \sqrt{N_{a t c}^{x}}+\epsilon_{a t c} \sqrt{N_{a t c}^{x}}
$$

Our conclusions remain intact when we obtain these estimates conditional on time (through splines of orthogonal polynomials over 6 years), cohort, and state effects. They are very similar to the results using unconditional estimates reported in the body of the paper.

Figure 22: Life-cycle profiles, males
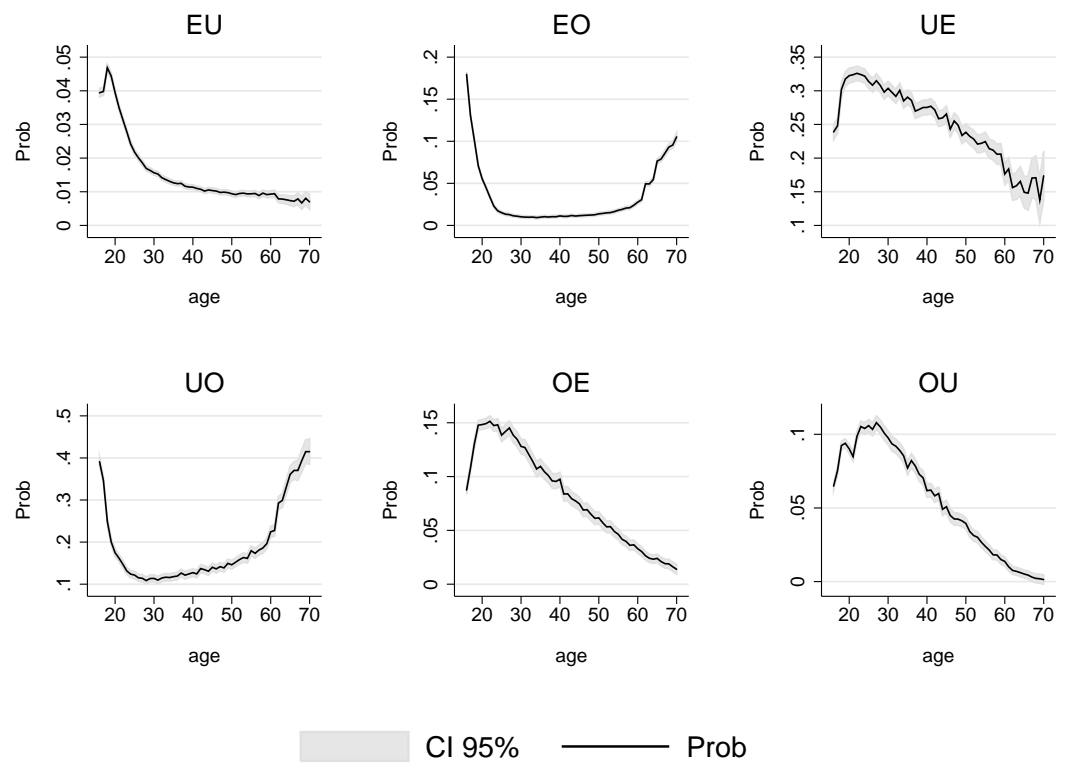

Note: Life-cycle profiles conditional on time, cohort and state estimated via Seemingly Unrelated Regressions (SUR). 
Figure 23: Life-cycle profiles, females
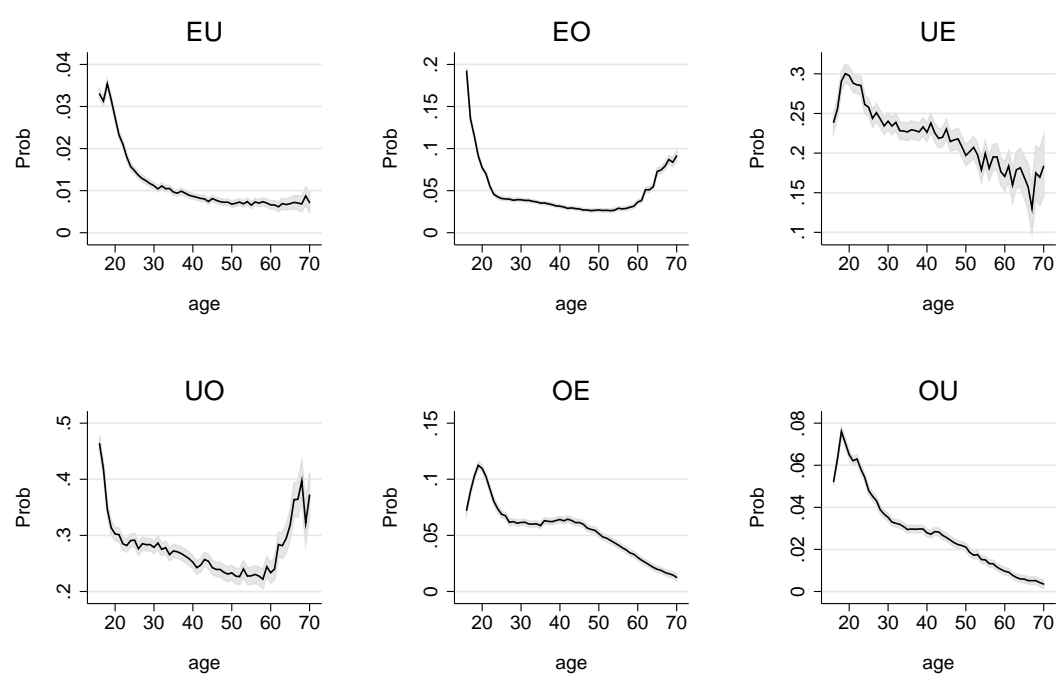

Cl $95 \%$ Prob

Note: Life-cycle profiles conditional on time, cohort and state estimated via Seemingly Unrelated Regressions (SUR).

Figure 24: Unemployment and Participation according to annual probabilities
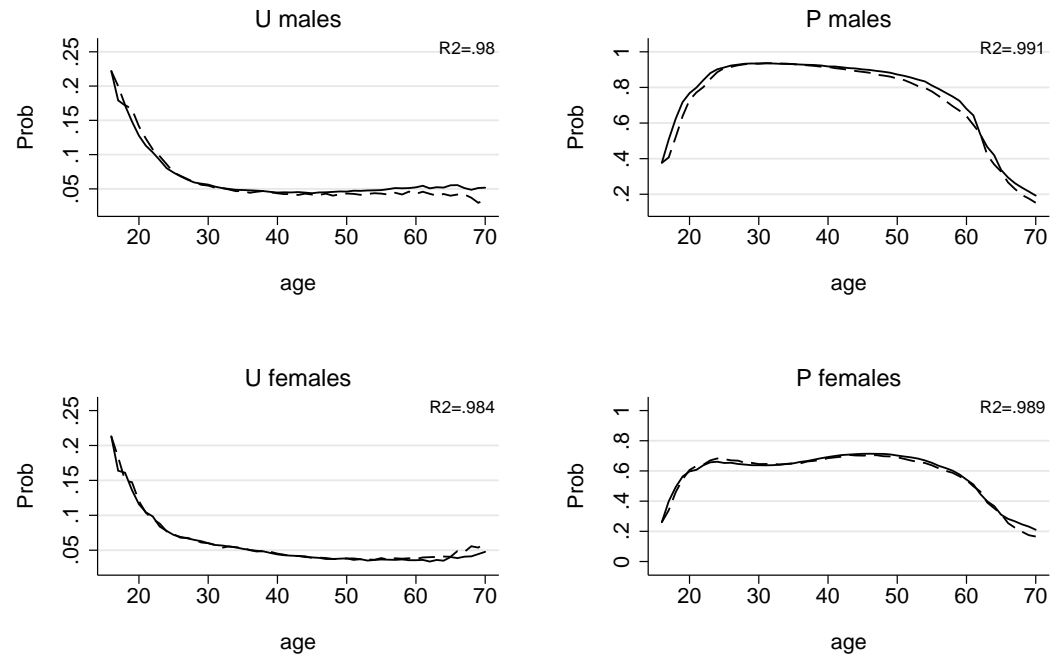

actual

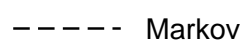

Note: Life-cycle profiles conditional on time, cohort and state estimated via Seemingly Unrelated Regressions (SUR). 
Figure 25: Importance of flows in Markov chains (AB1C): Unemployment, males
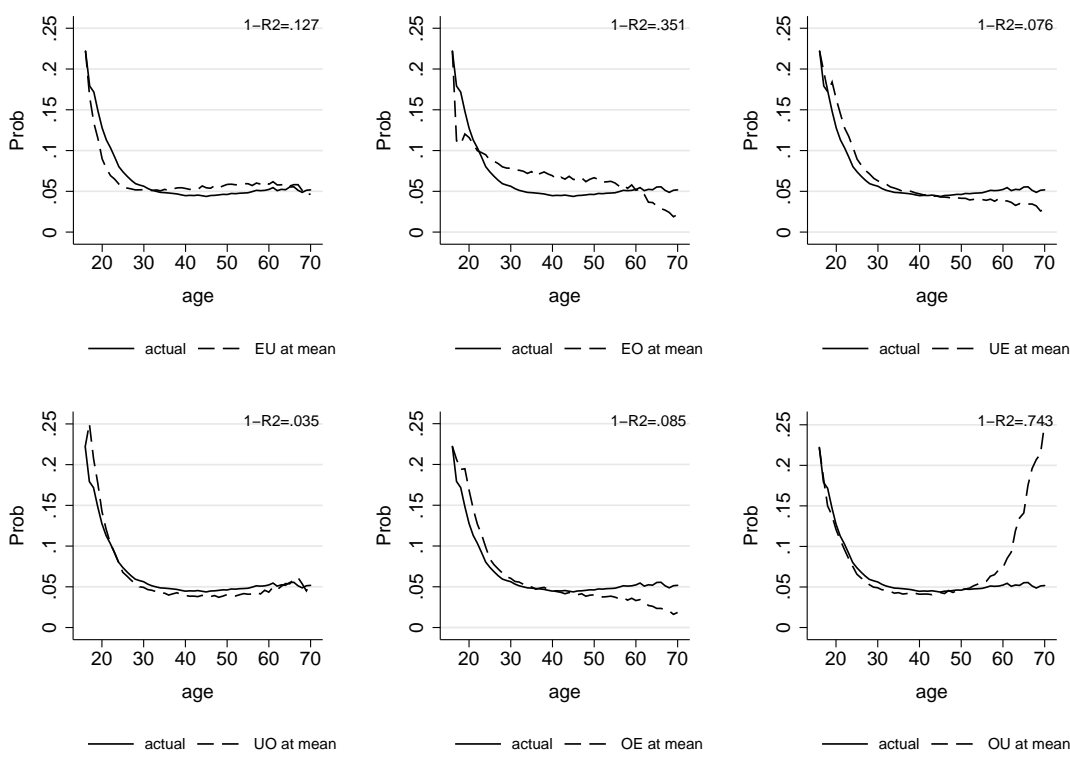

Note: Life-cycle profiles conditional on time, cohort and state estimated via Seemingly Unrelated Regressions (SUR).

Figure 26: Importance of flows in Markov chains (AB1C): Unemployment, females
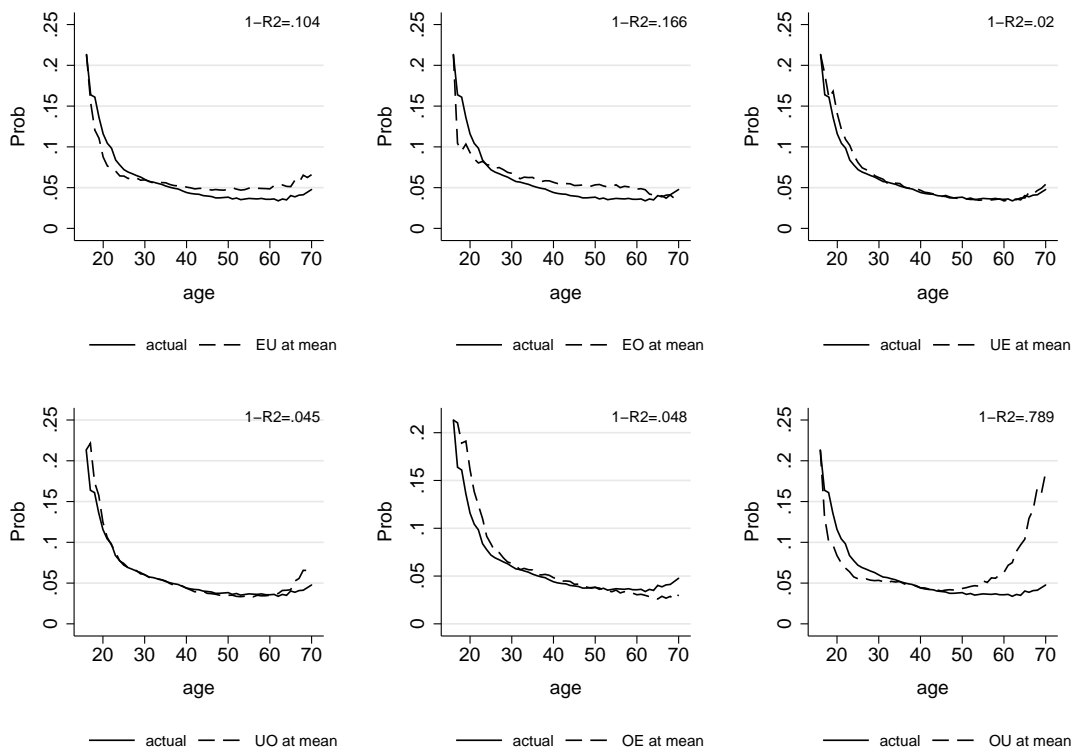

Note: Life-cycle profiles conditional on time, cohort and state estimated via Seemingly Unrelated Regressions (SUR). 
Figure 27: Importance of flows in Markov chains (AB1C): Participation, males
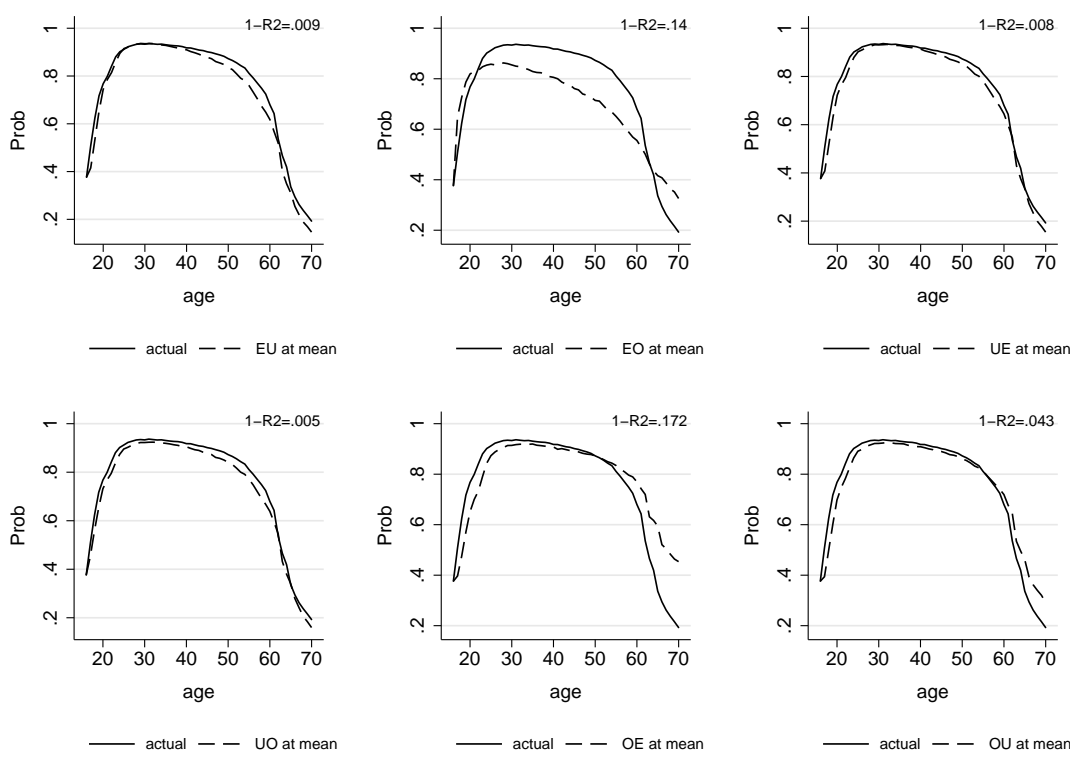

Note: Life-cycle profiles conditional on time, cohort and state estimated via Seemingly Unrelated Regressions (SUR).

Figure 28: Importance of flows in Markov chains (AB1C): Participation, females
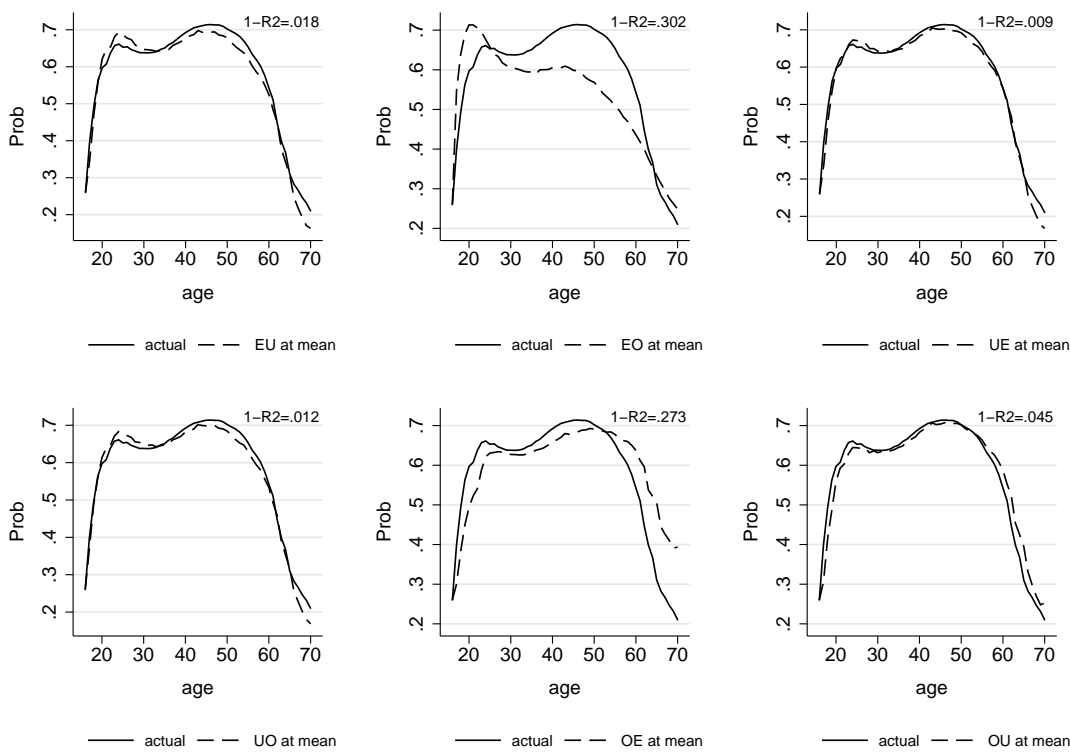

Note: Life-cycle profiles conditional on time, cohort and state estimated via Seemingly Unrelated Regressions (SUR). 
Figure 29: Variance decomposition by age group (AB1C): Unemployment
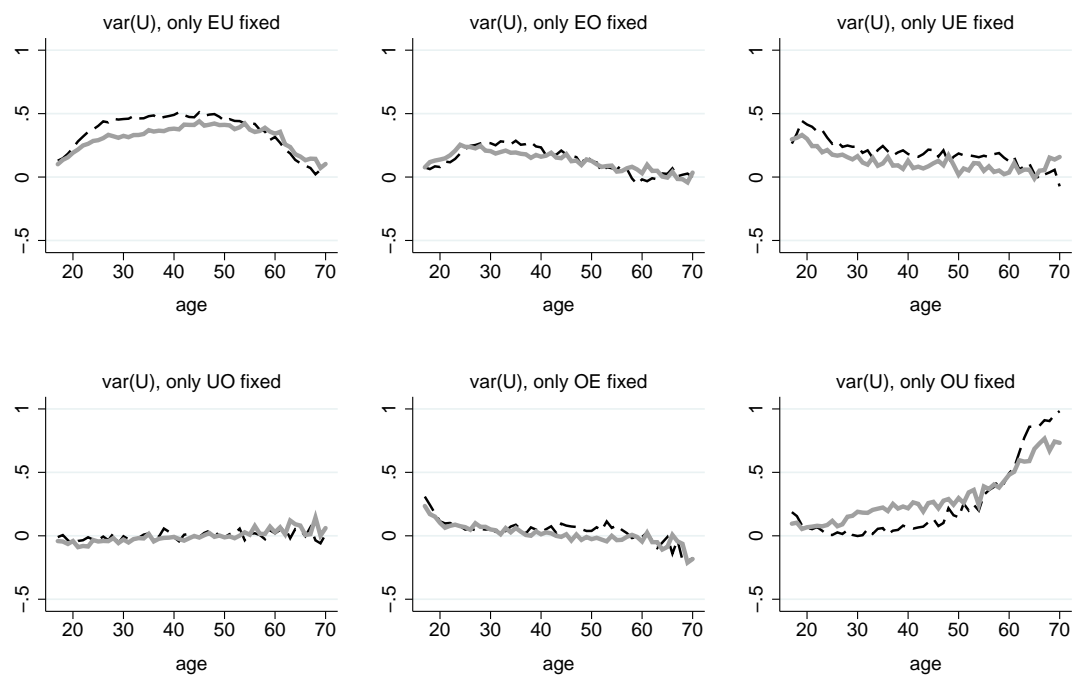

- - - male female

Note: Life-cycle profiles conditional on time, cohort and state estimated via Seemingly Unrelated Regressions (SUR). Figures show the percentage of $\operatorname{var}(\mathrm{U})$ explained by fixing one flow at a time, based on 10,000 simulations.

Figure 30: Variance decomposition by age group (AB1C): Participation
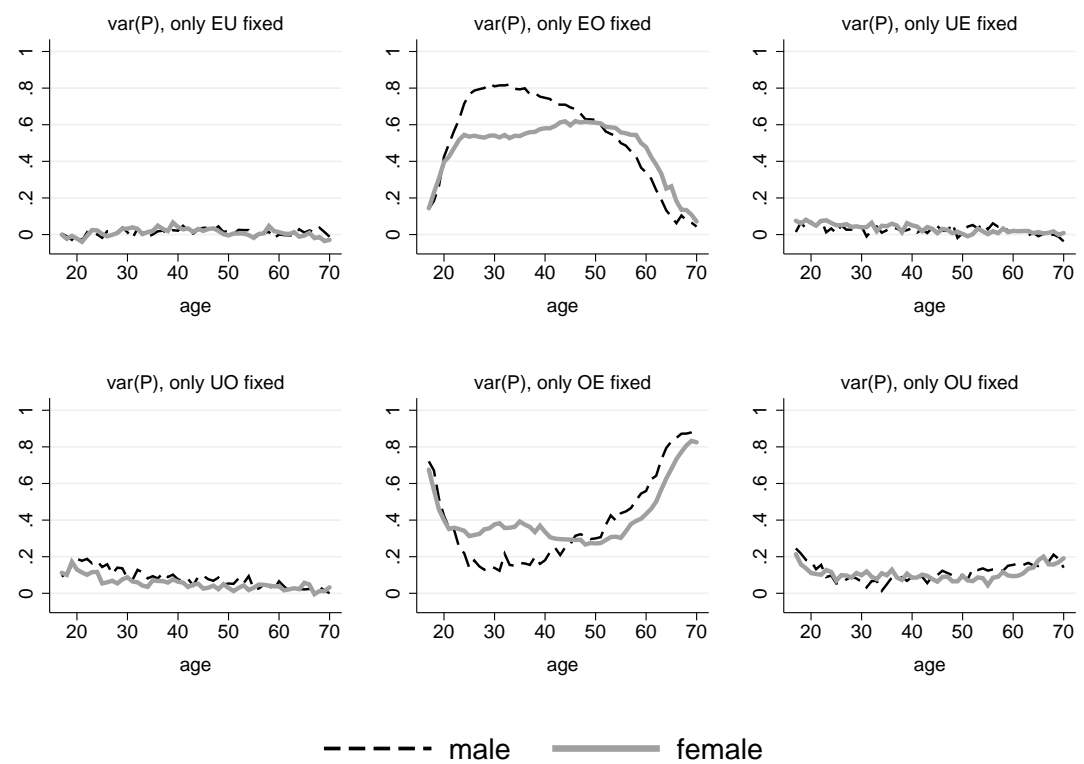

Note: Life-cycle profiles conditional on time, cohort and state estimated via Seemingly Unrelated Regressions (SUR). Figures show the percentage of $\operatorname{var}(\mathrm{U})$ explained by fixing one flow at a time, based on 10,000 simulations. 
Figure 31: Alternative Decomposition of life-cycle Unemployment rates, males
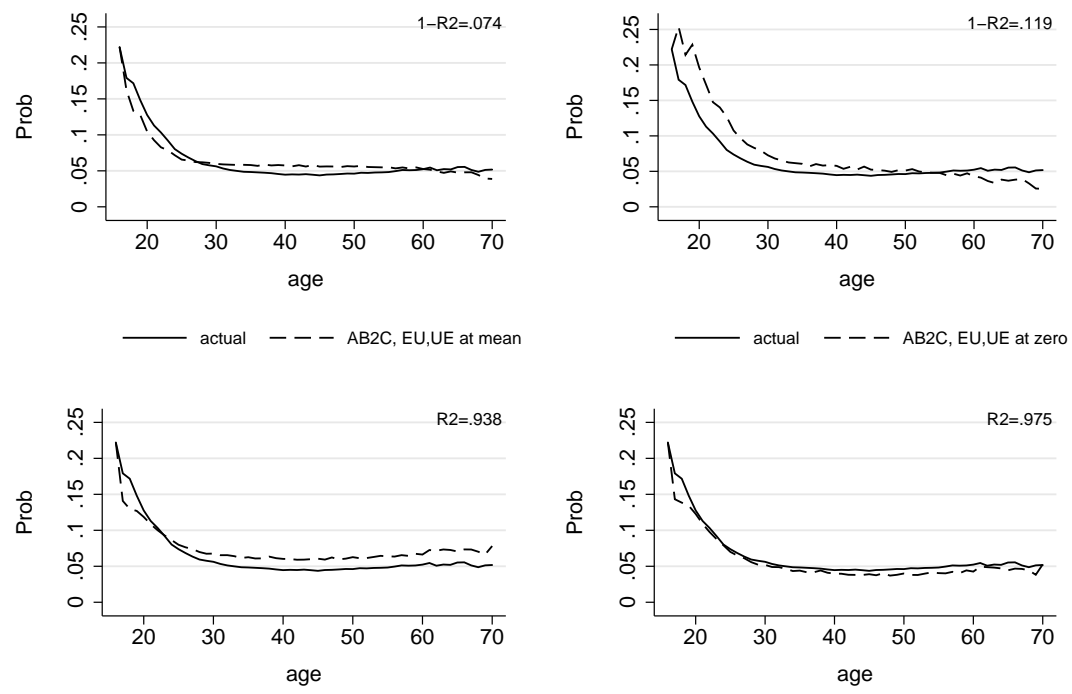

actual - - - AB2F at mean but EU,UE
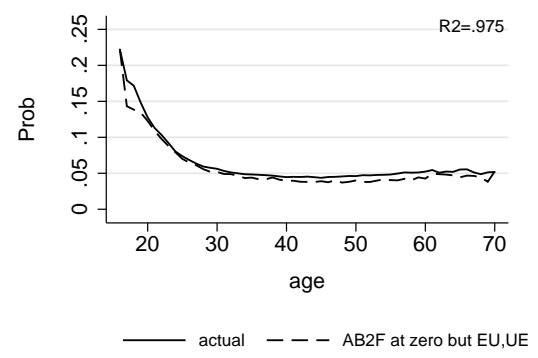

Note: Life-cycle profiles conditional on time, cohort and state estimated via Seemingly Unrelated Regressions (SUR).

Figure 32: Alternative Decomposition of life-cycle Unemployment rates, females
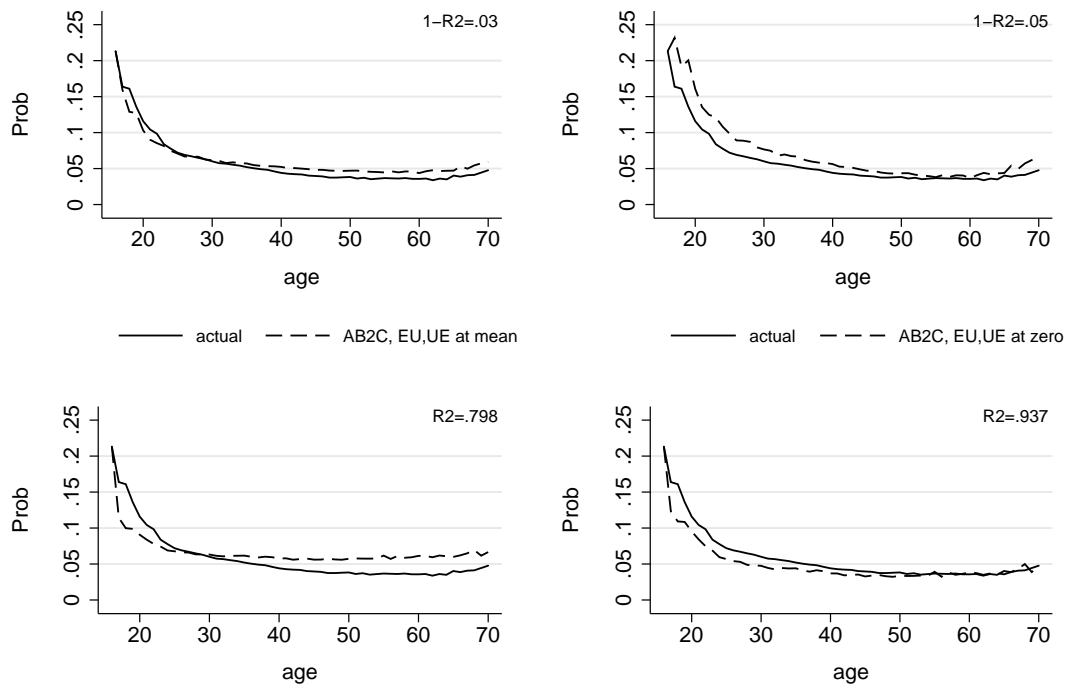

- actual --- AB2F a tmean but EU,UE

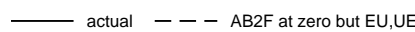

Note: Life-cycle profiles conditional on time, cohort and state estimated via Seemingly Unrelated Regressions (SUR). 
Figure 33: Ignoring inactivity: unemployment variance decomposition by age group

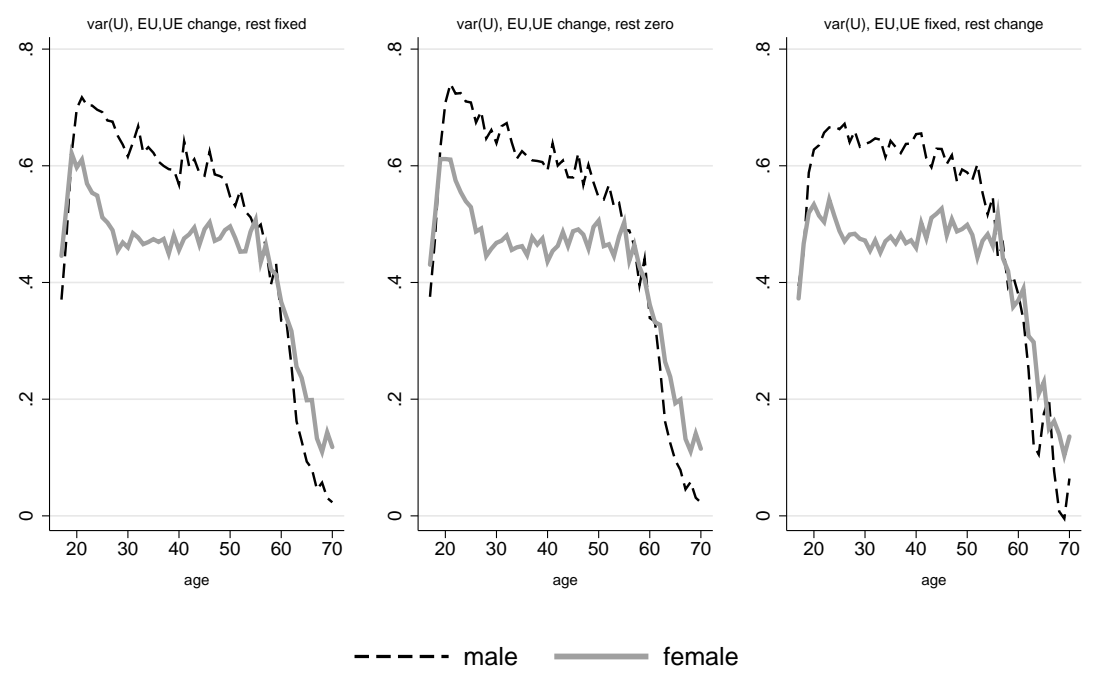

Note: Life-cycle profiles conditional on time, cohort and state estimated via Seemingly Unrelated Regressions (SUR). Each figure reports $\operatorname{var}(\mathrm{U})$ changes, based on 10000 simulations. 